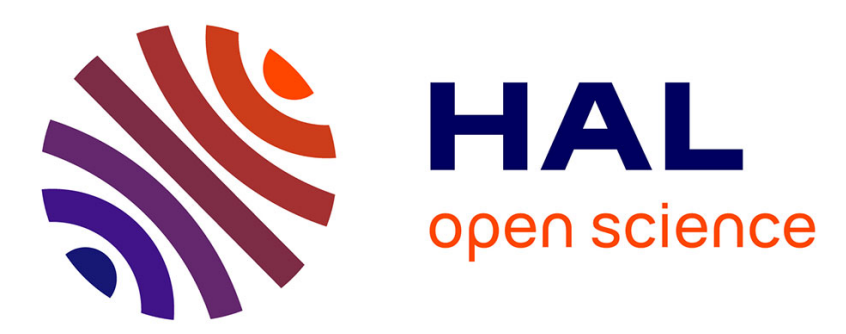

\title{
Dynamic behaviour of porous media saturated by a viscoelastic fluid. Application to bituminous concretes
}

\author{
C. Boutin, J.L. Auriault
}

\section{To cite this version:}

C. Boutin, J.L. Auriault. Dynamic behaviour of porous media saturated by a viscoelastic fluid. Application to bituminous concretes. International Journal of Engineering Science, 1990, 28 (11), pp.1157-1181. hal-00936259

\section{HAL Id: hal-00936259 \\ https://hal.science/hal-00936259}

Submitted on 24 Jan 2014

HAL is a multi-disciplinary open access archive for the deposit and dissemination of scientific research documents, whether they are published or not. The documents may come from teaching and research institutions in France or abroad, or from public or private research centers.
L'archive ouverte pluridisciplinaire HAL, est destinée au dépôt et à la diffusion de documents scientifiques de niveau recherche, publiés ou non, émanant des établissements d'enseignement et de recherche français ou étrangers, des laboratoires publics ou privés. 


\title{
DYNAMIC BEHAVIOUR OF POROUS MEDIA SATURATED BY A VISCOELASTIC FLUID. APPLICATION TO BITUMINOUS CONCRETES
}

\author{
C. BOUTIN ${ }^{1}$ and J. L. AURIAULT ${ }^{2}$ \\ ${ }^{1}$ Ecole Nationale des Travaux Publics de L'Etat, Laboratoire Géomatériaux, rue Maurice Audin, \\ 69518 Vaulx en Velin Cedex, France \\ ${ }^{2}$ Institut de Mecanique de Grenoble, UMR 101 (CNRS), Université Joseph Fourier, Institut National \\ Polytechnique de Grenoble, B.P. 53 X, 38041 Grenoble Cedex, France
}

(Communicated by R. M. BOWEN)

\begin{abstract}
This paper deals with the acoustics of porous media saturated by an incompressible viscous or viscoelastic fluid. By using the homogenization method for periodic structures, we determine the macroscopically equivalent medium. We show that the heterogeneous material displays three types of behaviour: biphasic, elastic, viscoelastic, according to the contrast of the mechanical properties of the constituants. This contrast is measured by the small parameter $\varepsilon$, ratio of the dimension of the pores to the macroscopic wave length. Therefore the macroscopic behaviour depends also on the excitation. The calculations of the effective macroscopic coefficients are performed for a one-dimensional geometry. The results applied to a porous media saturated by an Newtonian fluid show that the type of behaviour changes with the frequency. We treat the case of a saturation by a viscoelastic fluid by considering bituminous concretes. We investigate how this material behaves according to the temperature and the excitation frequency. The applicability of a time-temperature equivalency is studied and a comparison between experimental results and the 1D model is presented.
\end{abstract}

\section{INTRODUCTION}

This paper is devoted to the acoustics of the porous saturated media, with a more specific application to the bituminous concretes. This kind of heterogeneous material can be assimilied to a porous medium, constituted by the aggregates, saturated by the bitumen which is assumed to be an incompressible viscous or viscoelastic fluid. The mechanical properties of the bitumen present a variation of several decades when the temperature changes from the vitrification point $\left(\approx-15^{\circ} \mathrm{C}\right)$ to $150^{\circ} \mathrm{C}$. Consequently, the bituminous concrete shows different macroscopical behaviour, and we propose to analyse them here.

Numerous studies deal with the bituminous concrete. They are often developed in a phenomenological frame directly on the macroscopical scale, as in DiBenedetto [14] for the quasi-static behaviour, or in Huet [15] and Sayegh [20] for the dynamic behaviour. On this subject we can also note the more general theory of dynamics of porous saturated media, initialized by Biot [11]. Other authors treat the quasi-static behaviour by using the mixture theory: Atkin and Craine [1], Aubry et al. [3]. These varied approaches are limited because they introduce prerequisites for the description on the macroscopical scale. Thus, due to the modifications of the temperature or the excitation frequency, the different behaviour above mentioned cannot be analysed in a rigorous way.

In this work, despite the randomness of the media under consideration, we apply the homogenization method of heterogeneous periodic microstructures. Its aim is to derive the macroscopical equivalent continuum from the description of the physical mechanisms at the microscopic scale, Benssoussan et al. [10], Sanchez-Palencia [18]. No prerequisites are needed by this technique. The study of porous elastic media saturated by a Newtonian fluid display three kinds of macroscopic behaviour: biphasic, elastic, viscoelastic, according to the magnitude of the contrast between the mechanical properties of the constituants. On this topic, one can refer to Sanchez-Palencia [19] for the quasi-statics, and to Auriault [5], [8] for the dynamics. The main hypothesis of the method is to admit the existence of a small parameter $\varepsilon$ which is the ratio between the two characteristic lengths appearing in a dynamic problem in heterogeneous medium:

- on the one hand, the representative length $l$ of the microscopic periodic structure i.e. the pores; 
-on the other hand, the characteristic length $L$ at the macroscopic level. Concerning our study, we show that $L$ is connected to the macroscopical wave length $\lambda$ by the relation: $L=\lambda / 2 \pi$.

The great efficiency of the homogenization of periodic microstructures comes from the systematic use of $\varepsilon=l / L$ : Thus $\varepsilon$ is first used to quantify the contrasts between the properties of the constituants, and then to build the macroscopical description by means of asymptotical expansions in the power of $\varepsilon$, and last, as the measurement of the accuracy of the macroscopical description. By a rigorous procedure, we thus obtain the different kinds of behaviour according to the temperature of the heterogeneous medium and the frequency of the excitation.

In the first part we recall that the homogenization technique applied to the acoustics of elastic porous media saturated by a Newtonian fluid leads to three types of behaviour (biphasic, elastic, viscoelastic) depending on the contrast between the mechanical properties of the constituants. These results are then extended to the case of a linear viscoelastic saturating fluid. An application to a one-dimensional example is presented in part two. As the geometry is very simple, all the calculations can be treated analytically, so this example will be used as a reference in the last part.

As $L$ is directly in relation with the wave length, itself closely related with the frequency $f$, the macroscopical length $L$ depends on the problem studied. Consequently, the classification presented in the first part, based on the measurement by $\varepsilon$ of the contrast of the mechanical properties, must be improved because $\varepsilon$ depends on $L$ and therefore on the frequency. That is the purpose of the third part. In the fourth section we analyse the effects of the viscosity and the frequency, on the behaviour of a porous medium saturated by a Newtonian fluid.

Finally, in the last part we focus on the study of bituminous concretes: the influence of the temperature and of the frequency, the applicability of the principle of time-temperature equivalency at macroscopic level, and to end, a qualitative comparison between available experimental results and the monodimensional model are presented.

\section{HOMOgENIZATION OF A POROUS SATURATED MEDIUM}

All the homogenization processes are based on one fundamental hypothesis, without which no equivalent continuum medium can be obtained (Auriault and Caillerie [7]): the microscopic and macroscopic scales are very different: $\varepsilon=l / L \ll 1$. As a consequence, the material and the phenomena verify an invariancy property by spatial translation; here we choose to assume the microperiodicity of the material, $l$ being the dimension of the period.

Of course the bituminous concrete does not have a perfect periodic structure. But it seems that if the medium can be homogenized, the structure of the macroscopic law is independent of the stochastic or organized structure of the heterogeneities. [On the contrary, various examples show that it is not the case if $l=O(L)$.]

The two characteristic lengths $l$ and $L$, introduce two dimensionless space variables $X / l$ and $X / L$, their ratio being equal to $\varepsilon$. $A$ priori, the physical quantities, solutions to a given problem depend on these two dimensionless variables. (To simplify, we shall use the variables $x=X$ and $y=x / \varepsilon$, instead of $X / L$ and $X / l$.) We take into account the existence of the small parameter $\varepsilon$, introducing for the unknowns $\Phi$ an asymptotical expension in power of $\varepsilon$;

$$
\Phi(x, y)=\Phi^{0}(x, y)+\varepsilon \Phi^{1}(x, y)+\varepsilon^{2} \Phi^{2}(x, y)+\cdots
$$

Furthermore, the periodicity of the microstructure induces the same periodicity for the quantities $\Phi$ with regard to the variable $y$. So the functions $\Phi^{i}(x, y)$ are assumed to be periodic with respect to the variable $y$.

Finally, $\varepsilon$ is used to estimate the various dimensionless numbers associated to the problem. 


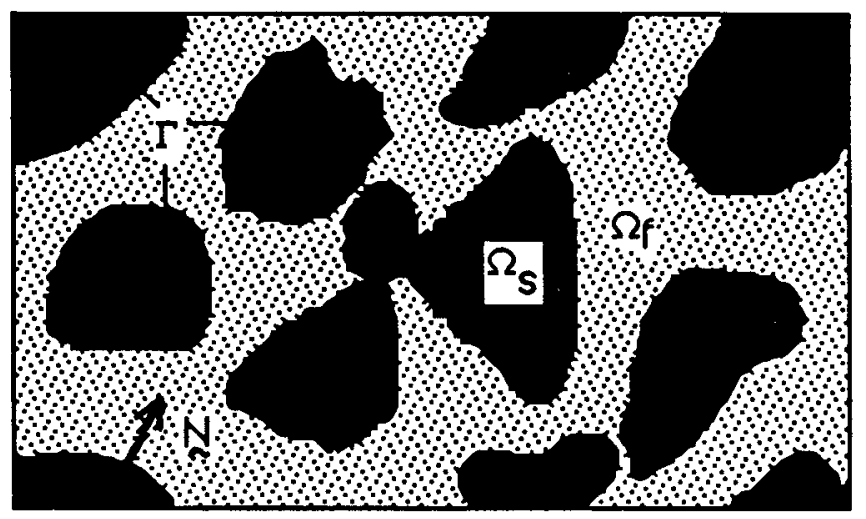

\section{Cell of a periodic porous medium}

Fig. 1. Cell of a periodic porous medium. $\Omega$, Volume of the period; $\Omega_{s}$, volume of the solid; $\Omega_{f}$, volume of the fluid; $\Gamma$, interface solid-fluid; $\mathbf{N}$, vector normal to $\Gamma ; \partial \Omega$, boundary of the cell.

\subsection{Description of the problem on the microscopic scale}

Let us consider a periodic porous saturated medium satisfying the following hypothesis:

-the volumes $\Omega_{\mathrm{s}}$ and $\Omega_{\mathrm{f}}$, respectively filled by the solid and the fluid, are connected;

- the skeleton is constituted by a linear elastic material;

- the saturating fluid is an incompressible viscous Newtonian fluid;

- the densities of the fluid $\rho_{\mathrm{f}}$ and the solid $\rho_{\mathrm{s}}$ have the same order of magnitude.

Moreover we assume that the strains of materials in the cell are small, and we consider that the fluid flow is in the linear range (the effects of the inertial convection are not taken into account.)

Finally we study harmonic excitations at frequency $f$ such as the wave diffraction on the heterogeneities can be neglected: $\lambda=2 \pi \cdot L \gg l$.

Under these conditions, the phenomena at the level of the period are governed by the following set of equations.

In the solid. The elastic constitutive law.

$$
\boldsymbol{\sigma}_{\mathrm{s}}=\mathbf{a} \cdot \mathbf{e}\left(\mathbf{U}_{\mathrm{s}}\right) ; \quad \sigma_{\mathrm{s} i j}=a_{i j k l} \cdot e_{k l}\left(\mathbf{U}_{\mathrm{s}}\right) ; \quad e_{k l}\left(U_{\mathrm{s}}\right)=\left(U_{\mathrm{s} k, l}+U_{\mathrm{s} l, k}\right) / 2
$$

The momentum balance.

$$
\operatorname{div}\left(\sigma_{\mathrm{s}}\right)=\rho_{\mathrm{s}} \cdot \partial^{2} \mathbf{U}_{\mathrm{s}} / \partial t^{2}=-\rho_{\mathrm{s}} \cdot \omega^{2} \mathbf{U}_{\mathrm{s}}
$$

Where $\mathbf{U}_{\mathrm{s}}$ is the solid displacement; $\mathbf{e}\left(\mathbf{U}_{\mathrm{s}}\right)$ the strain tensor; a the elastic tensor of the material constituting the skeleton; $\sigma_{\mathrm{s}}$ the stress tensor and $\omega$ the pulsation.

In the fluid. The viscous constitutive law.

$$
\sigma_{\mathrm{f}}=P \cdot \mathbf{I}+2 \cdot \mu \cdot \mathbf{D}(\mathbf{V}) \quad \text { (with the convention } P>0 \text { in traction) }
$$

The incompressibility.

$$
\operatorname{div}(\mathbf{V})=0
$$

The momentum balance (i.e. Navier-Stokes equation where the inertial convection is neglected).

$$
\operatorname{div}\left(\sigma_{\mathrm{f}}\right)=\operatorname{grad}(P)+\mu \cdot \Delta(\mathbf{V})=\rho_{\mathrm{f}} \cdot \partial \mathrm{V} / \partial t=\rho_{\mathrm{f}} \cdot i \omega \mathbf{V}
$$

With $\mathbf{V}$ the velocity of the fluid $\mathbf{V}=i \omega \cdot \mathbf{U}_{\mathrm{f}} ; \mathbf{U}_{\mathrm{f}}$ the fluid displacement;

$\mathbf{D}(\mathbf{V})$ the rate of strain tensor: $D_{k l}(\mathbf{V})=\left(V_{k, l}+V_{l, k}\right) / 2=i \omega \cdot e_{k l}\left(\mathbf{U}_{\mathrm{f}}\right)$; $\mu$ the dynamical viscosity; $\sigma_{\mathrm{f}}$ the stress tensor; $P$ the pressure. 
At the interface fluid-solid. The displacement continuity (adherence condition).

The normal stress continuity.

$$
\mathbf{U}_{\mathrm{f}}=\mathbf{U}_{\mathrm{s}}
$$

$$
\left(\sigma_{\mathrm{s}}-\boldsymbol{\sigma}_{\mathrm{f}}\right) \cdot \mathbf{N}=\mathbf{0}
$$

It appears that these equations involve the mechanical parameters of the two phases: $\rho_{\mathrm{s}}, \rho_{\mathrm{f}}$, $\mathbf{a}, \mu$; the pulsation $\omega$ of the excitation, and the variables $\mathbf{U}_{\mathrm{s}}, \mathbf{U}_{\mathrm{f}}, P$. Later on, we will consider that the elastic tensor a and the densities are given, but we keep $\mu, \omega$ as parameters and $\mathbf{U}_{\mathrm{s}}$, $\mathbf{U}_{\mathrm{f}}, P$ as variables. Thus the physics of the problem is governed by four independent variable dimensionless numbers, connecting these five quantities.

\subsection{Dimensionless numbers}

In the solid. Let us introduce the number $T_{\mathrm{s}}=\rho_{\mathrm{s}} \cdot \omega^{2}\left|\mathbf{U}_{\mathrm{s}}\right| /\left|\operatorname{div}\left(\sigma_{\mathrm{s}}\right)\right|$ measuring the ratio between the inertial and the internal elastic forces. Using $l$ as reference length, and evaluating $T_{\mathrm{s} l}$ at the microscopic scale, we get: $T_{s} l \approx \rho_{\mathrm{s}} \cdot \omega^{2} l^{2} /|\mathbf{a}|$. This relation can be interpreted in two different ways:

$T_{\mathrm{s} l}=\left(l / \lambda_{\mathrm{s}}\right)^{2}$ where $\lambda_{\mathrm{s}}=\omega \sqrt{|\mathbf{a}| / \rho_{\mathrm{s}}}$ is the characteristic wave length in the elastic constituent.

$T_{\mathrm{s} l}=\left(\omega \cdot \tau_{\mathrm{s}}\right)^{2}$ where $\tau_{\mathrm{s}}=l \cdot \sqrt{\rho_{\mathrm{s}} /|\mathbf{a}|}$ is the time needed by the wave to cross the period.

In all the cases examined, the magnitude of $T_{\mathrm{s} l}$ will be taken as equal to $\varepsilon^{2}$. It can be shown that if $T_{\mathrm{sl}}>O\left(\varepsilon^{2}\right)$ the phenomena are not homogenizable, i.e. an equivalent macroscopical description cannot be found; and if $T_{\mathrm{s} l}<O\left(\varepsilon^{2}\right)$ the problem becomes quasi-static [8].

In the fluid. As we have to distinguish the effects of the pressure and the one of the viscous stresses, we define two dimensionless numbers:

$T_{\mathrm{fl}}=\rho_{\mathrm{f}} \cdot|\partial \mathrm{V} / \partial t| /|\operatorname{grad}(P)| \approx \rho_{\mathrm{f}} \cdot l \cdot \omega^{2}\left|\mathbf{U}_{\mathrm{f}}\right| / P$. For similar reasons to those concerning the solid phase, we consider in all the cases that $T_{\mathrm{f} l}=O(\varepsilon)$.

$R T_{l}=\rho_{\mathrm{f}} \cdot|\partial \mathbf{V} / \partial t| /|\mu \cdot \Delta(\mathbf{V})| \approx \rho_{\mathrm{f}} \cdot \omega \cdot l^{2} / \mu . R T_{l}$ is the transient Reynolds number which can be written also as: $R T_{l}=\left(l / \lambda_{\mathrm{f}}\right)^{2}=\left(\omega \cdot \tau_{\mathrm{f}}\right)^{2}$ where:

$\lambda_{\mathrm{f}}=\sqrt{\mu / \omega \rho_{\mathrm{f}}}$ is the thickness of the boundary layer appearing at the fluid-solid interface.

$\tau_{\mathrm{f}}=l \cdot \sqrt{\rho_{\mathrm{f}} / \mu \omega}$ is the time needed by the diffusive shear-wave to cross the period.

This last dimensionless number has a great influence on the macroscopic description, and we will investigate the following three cases of interest: $R T_{l}=O(1), O(\varepsilon), O\left(\varepsilon^{2}\right)$.

At the fluid-solid interface. The displacement continuity leads us to assume that in all the cases $U=\left|\mathbf{U}_{\mathrm{s}}\right| /\left|\mathbf{U}_{\mathrm{f}}\right|=O(1)$.

In conclusion we have defined four independent dimensionless numbers associated to the five variable quantities, that is to say $\mathbf{U}_{\mathrm{s}}, \mathbf{U}_{\mathrm{f}}, P, \omega, \mu$. In the next section, we homogenize the medium under the conditions above mentioned (where $l$ was used as a characteristic normalization length).

Remarks

- The comparison between the stresses in the fluid and in the solid can be made using the above dimensionless numbers:

For example, the ratio between the pressure and the elastic stress is given by:

$$
P /\left|\mathbf{a} \cdot \mathbf{e}\left(\mathbf{U}_{\mathrm{s}}\right)\right| \approx P \cdot l /|\mathbf{a}| \cdot\left|\mathbf{U}_{\mathrm{s}}\right|=\left(T_{\mathrm{s} l} / T_{\mathrm{f} l}\right) \cdot\left(\rho_{\mathrm{f}} / \rho_{\mathrm{s}}\right) \cdot U=O(\varepsilon)
$$

Likewise, the ratio between the viscous and elastic stresses is equal to:

$$
\mu \cdot|\mathbf{D}(\mathbf{V})| /\left|\mathbf{a} \cdot \mathbf{e}\left(\mathbf{U}_{\mathrm{s}}\right)\right| \approx(\mu \omega /|\mathbf{a}|) \cdot\left(\left|\mathbf{U}_{\mathrm{f}}\right| /\left|\mathbf{U}_{\mathrm{s}}\right|\right)=U \cdot T_{\mathrm{s}} / R T
$$

and will then be of a magnitude of $O\left(\varepsilon^{2}\right), O(\varepsilon), O(1)$.

-it is important to note that with the previous assumptions, the measurement of $R T$ is directly connected to the contrast of the "moduli" of the fluid $(\mu \omega)$ and the solid (a)

-In order to specify the physics at the microscopic level it was convenient to use $l$ as a characteristic normalization length. On the contrary, when looking at the macroscopic behaviour, it is more suitable to take $L$ as a characteristic normalization length. Then 
the magnitude of the dimensionless numbers becomes:

$$
T_{\mathrm{s} L}=O(1), \quad T_{\mathrm{f} L}=O(1), \quad U=O(1), \quad R T_{L}=O\left(\varepsilon^{-2}\right), O\left(\varepsilon^{-1}\right), O(1) .
$$

Later on we will prefer to use these values.

\subsection{Homogenization of a porous medium saturated by a Newtonian fluid}

In this section we briefly recall the research carried out by Auriault [4], [5], [8], references that the reader is invited to consult for more precise details. These results are the basis of our study of porous media saturated by a viscoelastic fluid. The method consists of introducing the above presented asymptotic developments for $\Phi$, into the equations describing the physics at the local scale:

$$
\Phi(x, y)=\Phi^{0}(x, y)+\varepsilon \Phi^{1}(x, y)+\varepsilon^{2} \Phi^{2}(x, y)+\cdots
$$

Using such a process, where $x$ is the driving space variable, implies that the local description is normalized with the macroscopic length $L$, i.e. the dimensionless numbers in consideration are $T_{\mathrm{s} L}, T_{\mathrm{f} L}, R T_{L}$. And the spacial derivation $\partial / \partial X$, becomes: $\partial / \partial x+\varepsilon^{-1} \partial / \partial y$.

After which, like powers of $\varepsilon$ lead to boundary value problems which have to be solved, for the different terms of the developments. The macroscopic description follows.

2.3.1 Biphasic macroscopic behaviour. $R T_{L}=O\left(\varepsilon^{-2}\right)$.

Let us study the case where at the macroscopic level: $T_{\mathrm{s} L}=O(1), T_{\mathrm{f} L}=O(1), U=O(1)$, $R T_{L}=O\left(\varepsilon^{-2}\right)$. Note that these values are reached as soon as $\mu \omega /|\mathbf{a}|=O\left(\varepsilon^{2}\right)$.

Equations (1)-(7) describe the medium, but in order to take into account the magnitude of $R T_{L}$, equation (5) is formally modified to:

$$
\operatorname{grad}(P)+\varepsilon^{2} \cdot \mu \cdot \Delta(\mathbf{V})=\rho_{\mathrm{f}} \cdot i \omega \mathrm{V}
$$

According to these conditions, the homogenization is performed as follows:

Zero order for the solid displacement and the fluid pressure. The problem to be solved for the solid displacement $\mathbf{U}_{\mathrm{s}}^{0}$ is given by the $\varepsilon^{-2}$ terms in equation (2) and the $\varepsilon^{-1}$ terms in equation (7):

$$
\mid \begin{array}{ll}
\mathbf{U}_{\mathrm{s}}^{0}(x, y) & \Omega \text {-periodic in } y . \\
\operatorname{div}_{y}\left(\mathbf{a} \cdot \mathbf{e}_{y}\left(\mathbf{U}_{\mathrm{s}}^{0}\right)\right)=0 & \text { in } \Omega_{\mathrm{s}} \\
\left(\mathbf{a} \cdot \mathbf{e}_{y}\left(\mathbf{U}_{\mathrm{s}}^{0}\right)\right) \cdot \mathbf{N}=0 & \text { on } \Gamma
\end{array}
$$

This is an homogeneous differential problem with respect to the $y$ variable; its obvious solution is:

$$
\mathbf{U}_{\mathrm{s}}^{0}(x, y)=\mathbf{U}_{\mathrm{s}}^{0}(x)
$$

Concerning the pressure, equation $\left(5^{*}\right)$ at $\varepsilon^{-1}$ order gives $\operatorname{grad}_{y}\left(P^{0}\right)=0$. Thus $P^{0}(x, y)=$ $P^{0}(x)$.

Consequently, at zero order, the solid displacement and the pressure are independent of the local variable $y$.

First order for the solid displacement. The determination of $\mathbf{U}_{\mathrm{s}}^{1}$ is achieved by equation (2) at $\varepsilon^{-1}$ order and (7) at zero order:

$$
\mid \begin{array}{ll}
\mathbf{U}_{\mathrm{s}}^{1}(x, y) & \Omega \text {-periodic in } y . \\
\operatorname{div}_{y}\left(\mathbf{a}\left(\mathbf{e}_{y}\left(\mathbf{U}_{\mathrm{s}}^{1}\right)+\mathbf{e}_{x}\left(\mathbf{U}_{\mathrm{s}}^{0}\right)\right)\right)=0 & \text { in } \boldsymbol{\Omega}_{\mathrm{s}} \\
\left(\mathbf{a} \cdot\left(\mathbf{e}_{y}\left(\mathbf{U}_{\mathrm{s}}^{1}\right)+\mathbf{e}_{x}\left(\mathbf{U}_{\mathrm{s}}^{0}\right)\right)\right) \cdot \mathbf{N}=\boldsymbol{P}^{0} \cdot \mathbf{N} & \text { on } \Gamma
\end{array}
$$

Except for an additive constant, the variational formulation corresponding to this problem is as follows:

$$
\forall \mathbf{W} \in E, \quad \int_{\Omega_{s}} \mathbf{a} \cdot \mathbf{e}_{y}\left(\mathbf{U}_{\mathrm{s}}^{1}\right) \cdot \mathbf{e}_{y}(\mathbf{W}) \cdot \mathrm{d} \Omega=-\int_{\Omega_{\mathrm{s}}}\left(\mathbf{a} \cdot \mathbf{e}_{x}\left(\mathbf{U}_{\mathrm{s}}^{0}\right) \cdot \mathbf{e}_{y}(\mathbf{W})-P^{0} \cdot \operatorname{div}_{y}(\mathbf{W})\right) \cdot \mathrm{d} s
$$

Where the space $E$ of the $\Omega$-periodic vectors $\mathbf{W}$, defined on $\Omega_{\mathrm{s}}$, having a null average on the cell, is associated with the appropriate scalar product. Because of the Lax-Milgram lemma, the existence and the uniqueness of the solution is ensured. 
The linearity of the problem leads to:

$$
U_{\mathrm{s} i}^{1}=u_{\mathrm{si}}^{1}(x)+\xi_{i j k} \cdot e_{x j k}\left(\mathbf{U}_{\mathrm{s}}^{0}\right)+\eta_{i} \cdot P^{0}
$$

the vectors $\eta(y)$ and $\xi_{i k}(y)$ being the particular solutions of (9) for:

$$
\begin{array}{lll}
P^{0}=1, & e_{x j k}\left(\mathbf{U}_{\mathrm{s}}^{0}\right)=0, \quad \text { and } \\
P^{0}=0, & e_{x l m}\left(\mathbf{U}_{\mathrm{s}}^{0}\right)=\left(\delta_{j l} \cdot \delta_{k m}+\delta_{j m} \cdot \delta_{k l}\right) / 2 \quad \text { respectively. }
\end{array}
$$

Zero order for the fluid displacement (first order for the pressure). Equations $\left(5^{*}\right)$ at zero order, (4) at $\varepsilon^{-1}$ order, (6) at zero order, lead to the system below allowing the determination of $\mathbf{U}_{\mathbf{f}}^{0}$ and $P^{1}$ :

$$
\mid \begin{array}{ll}
P^{1}(x, y) \text { and } \mathbf{U}_{\mathrm{f}}^{0}(x, y) & \Omega \text {-periodic in } y \\
\operatorname{grad}_{x}\left(P^{0}\right)+\operatorname{grad}_{y}\left(P^{1}\right)+i \omega \mu \cdot \Delta_{y}\left(\mathbf{U}_{\mathrm{f}}^{0}\right)=-\rho_{\mathrm{f}} \cdot \omega^{2} \mathbf{U}_{\mathrm{f}}^{0} & \text { in } \Omega_{\mathrm{f}} \\
\operatorname{div}_{\mathbf{y}}\left(\mathbf{U}_{\mathrm{f}}^{0}\right)=0 & \text { in } \Omega_{\mathrm{f}} \\
\mathbf{U}_{\mathrm{f}}^{0}=\mathbf{U}_{\mathrm{s}}^{0} & \text { on } \Gamma
\end{array}
$$

Since $\mathbf{U}_{\mathrm{s}}^{0}=\mathbf{U}_{\mathrm{s}}^{0}(x)$, putting down $\mathbf{W}^{0}=\mathbf{U}_{\mathrm{f}}^{0}-\mathbf{U}_{\mathrm{s}}^{0}$, we obtain:

$$
\mid \begin{array}{ll}
P^{1}(x, y) \text { and } \mathbf{W}^{0}(x, y) & \Omega \text {-periodic in } y . \\
\operatorname{grad}_{y}\left(P^{1}\right)+i \omega \mu \cdot \Delta_{y}\left(\mathbf{W}^{0}\right)+\rho_{\mathrm{f}} \cdot \omega^{2} \mathbf{W}^{0}=-\operatorname{grad}_{x}\left(P^{0}\right)-\rho_{\mathrm{f}} \cdot \omega^{2} \mathbf{U}_{\mathrm{s}}^{0} & \text { in } \Omega_{\mathrm{f}} \\
\operatorname{div}_{y}\left(\mathbf{W}^{0}\right)=0 & \text { in } \Omega_{\mathrm{f}} \\
\mathbf{W}^{0}=0 & \text { on } \Gamma
\end{array}
$$

The variational formulation of this problem is:

$\forall \mathbf{W} \in E^{\prime}$,

$$
\int_{\Omega_{\mathrm{f}}}\left(i \omega \cdot \mu \cdot \mathbf{e}_{y}\left(\mathbf{W}^{0}\right) \cdot \mathbf{e}_{y}(\mathbf{W})+\rho_{\mathrm{f}} \cdot \omega^{2} \mathbf{W}^{0} \cdot \mathbf{W}\right) \cdot \mathrm{d} \Omega=-\int_{\Omega_{\mathrm{f}}} \mathbf{W} \cdot\left(\operatorname{grad}_{x}\left(P^{0}\right)+\rho_{\mathrm{f}} \cdot \omega^{2} \mathbf{U}_{\mathrm{s}}^{0}\right) \mathrm{d} \Omega
$$

where the space $E^{\prime}$ of complex $\Omega$-periodic vectors $W$, defined on $\Omega_{\mathrm{f}}$, null on $\partial \Omega$, is associated with the appropriate hermitian product. Once more the variational formulation above mentioned proves the existence and the uniqueness of $W^{0}$ and the linearity leads to:

$$
i \omega \mu W_{i}^{0}=i \omega \mu \cdot\left(\mathbf{U}_{\mathrm{f}}^{0}-\mathbf{U}_{\mathrm{s}}^{0}\right)_{i}=\left(k_{i j}\right) \cdot\left(\operatorname{grad}_{x}\left(P^{0}\right)+\rho_{\mathrm{f}} \cdot \omega^{2} \mathbf{U}_{\mathrm{s}}^{0}\right)_{j}
$$

$k_{i} / i \omega \mu$ being the complex $\omega$-dependent displacement $W^{0}$, solution of (12) when:

$$
\left(\operatorname{grad}_{x}\left(P^{0}\right)+\rho_{\mathrm{f}} \cdot \omega^{2} \mathbf{U}_{\mathrm{s}}^{0}\right)_{j}=\delta_{i j}
$$

Higher order: compatibility equations. Contrary to the previous problems, the differential systems concerning the higher orders, does not necessarily have a solution. Therefore, in order to ensure the existence of the following terms in the asymptotic expansions, we have to introduce additional equations, so-called compatibility equations. These compatibility equations concern the momentum and volume balances.

Momentum balance. Regarding equations (2) and (5) at zero order and (7) at first order, we obtain the following set of equations:

$$
\mid \begin{array}{ll}
\sigma_{\mathrm{s}}^{1}(x, y) \quad \text { and } \quad \sigma_{\mathrm{f}}^{1}(x, y) & \Omega \text {-periodic in } y \\
\operatorname{div}_{y}\left(\sigma_{\mathrm{s}}^{1}\right)+\operatorname{div}_{x}\left(\sigma_{\mathrm{s}}^{0}\right)=-\rho_{\mathrm{s}} \cdot \omega^{2} \mathbf{U}_{\mathrm{s}}^{0} & \text { in } \Omega_{\mathrm{s}} \\
\left(\sigma_{\mathrm{s}}^{1}-\sigma_{\mathrm{f}}^{1}\right) \cdot \mathbf{N}=0 & \text { on } \Gamma \\
\operatorname{div}_{y}\left(\sigma_{\mathrm{f}}^{1}\right)+\operatorname{div}_{x}\left(\sigma_{\mathrm{f}}^{0}\right)=-\rho_{\mathrm{f}} \omega^{2} \mathbf{U}_{\mathrm{f}}^{0} & \text { in } \Omega_{\mathrm{f}}
\end{array}
$$

Putting down:

$$
\sigma^{1} \mid \begin{array}{lll}
=\sigma_{\mathrm{s}}^{1}(x, y) & \text { in } \Omega_{\mathrm{s}} & \boldsymbol{\sigma}^{0} \mid \begin{array}{ll}
=\boldsymbol{\sigma}_{\mathrm{s}}^{0}(x, y)=\mathbf{a} \cdot\left(\mathbf{e}_{y}\left(\mathbf{U}_{\mathrm{s}}^{1}\right)+\mathbf{e}_{x}\left(\mathbf{U}_{\mathrm{s}}^{0}\right)\right) & \text { in } \Omega_{\mathrm{s}} \\
=\sigma_{\mathrm{f}}^{1}(x, y) & \text { in } \Omega_{\mathrm{f}}
\end{array} \quad \text { in } \Omega_{\mathrm{f}} .
\end{array}
$$

The continuity and periodicity conditions imply:

$$
\int_{\Omega} \operatorname{div}_{y}\left(\sigma^{1}\right) \cdot \mathrm{d} \Omega=0 .
$$


Therefore the above problem has a solution only if:

$$
-\int_{\Omega} \operatorname{div}_{x}\left(\sigma^{0}\right) \cdot \mathrm{d} \Omega=\rho_{\mathrm{s}} \cdot \omega^{2} \int_{\Omega_{\mathrm{s}}} \mathbf{U}_{\mathrm{s}}^{0} \cdot \mathrm{d} \Omega+\rho_{\mathrm{f}} \cdot \omega^{2} \int_{\mathbf{\Omega}_{\mathrm{f}}} \mathbf{U}_{\mathrm{f}}^{0} \cdot \mathrm{d} \Omega .
$$

It has been pointed out that this condition is also sufficient [5]; so a first compatibility equation is:

$$
\operatorname{div}_{x}\left\langle\sigma^{0}\right\rangle=-(1-n) \cdot \rho_{\mathrm{s}} \cdot \omega^{2} \mathbf{U}_{\mathrm{s}}^{0}-n \cdot \rho_{\mathrm{f}} \cdot \omega^{2}\left\langle\mathbf{U}_{\mathrm{f}}^{0}\right\rangle
$$

where $\langle\cdot\rangle=|\Omega|^{-1} \int_{\Omega} \cdot \mathrm{d} \Omega ; n=\left|\Omega_{\mathrm{f}}\right| /\left|\Omega_{\mathrm{s}}\right|$ is the porosity, and, for the sake of simplicity, we assume $\rho_{\mathrm{s}}$ to be $y$-independent.

Volume balance. Regarding the fluid incompressibility (4) and the adherence condition (6), respectively at the orders zero and one, we have:

$$
\mid \begin{array}{ll}
\mathbf{U}_{\mathrm{f}}^{1}(x, y) \text { and } \mathbf{U}_{\mathrm{s}}^{1}(x, y) & \Omega \text { periodic in } y . \\
\operatorname{div}_{y}\left(\mathbf{U}_{\mathrm{f}}^{1}\right)+\operatorname{div}_{x}\left(\mathbf{U}_{\mathrm{f}}^{0}\right)=0 & \text { in } \Omega_{\mathrm{f}} \\
\mathbf{U}_{\mathrm{f}}^{1}=\mathbf{U}_{\mathrm{s}}^{1} & \text { in } \Gamma
\end{array}
$$

As above the continuity and periodicity conditions imply that:

$$
\int_{\Omega_{\mathrm{f}}} \operatorname{div}_{y}\left(\mathbf{U}_{\mathrm{f}}^{1}\right) \cdot \mathrm{d} \Omega+\int_{\Omega_{\mathrm{s}}} \operatorname{div}_{y}\left(\mathbf{U}_{\mathrm{s}}^{1}\right) \cdot \mathrm{d} \Omega=0
$$

which leads us to a second compatibility equation:

$$
\operatorname{div}_{x}\left\langle\mathbf{U}_{\mathbf{f}}^{0}\right\rangle=\int_{\Omega_{\mathrm{s}}} \operatorname{div}_{y}\left(\mathbf{U}_{\mathrm{s}}^{1}\right) \cdot \mathrm{d} \Omega
$$

Equivalent macroscopic behaviour. Substituting $\mathbf{U}_{\mathbf{s}}^{1}$ by its expression (10) in the compatibility equations, we obtain the equivalent macroscopic behaviour (where, for more simplicity, the exponent ${ }^{0}$ and the index $\boldsymbol{x}$ for the derivatives have been omitted):

with

$$
\mid \begin{aligned}
& \langle\boldsymbol{\sigma}\rangle=\mathbf{C} \cdot \mathbf{e}\left(\mathbf{U}_{\mathrm{s}}\right)+\alpha \cdot P \\
& \operatorname{div}\langle\boldsymbol{\sigma}\rangle=-(1-n) \cdot \rho_{\mathrm{s}} \cdot \omega^{2} \mathbf{U}_{\mathrm{s}}-n \cdot \rho_{\mathrm{f}} \cdot \omega^{2}\left\langle\mathbf{U}_{\mathrm{f}}\right\rangle \\
& i \omega\left(\left\langle\mathbf{U}_{\mathrm{f}}\right\rangle-n \cdot \mathbf{U}_{\mathrm{s}}\right)=(\mathbf{K} / \mu)\left(\operatorname{grad}(P)+\rho_{\mathrm{f}} \cdot \omega^{2} \mathbf{U}_{\mathrm{s}}\right) \\
& \operatorname{div}\left(\left\langle\mathbf{U}_{\mathrm{f}}\right\rangle-n \cdot \mathbf{U}_{\mathrm{s}}\right)=-\alpha \cdot \mathbf{e}\left(\mathbf{U}_{\mathrm{s}}\right)+\beta \cdot P
\end{aligned}
$$

$$
\begin{array}{ll}
C_{i j k l}=\left\langle a_{i j k l}+a_{i j m n} \cdot e_{y m n}\left(\xi_{k l}\right)\right\rangle & \beta=\left\langle\operatorname{div}_{y}(\boldsymbol{\eta})\right\rangle \\
\alpha_{i j}=n \cdot \delta_{i j}+\left\langle a_{i j m n} \cdot e_{y m n}(\boldsymbol{\eta})\right\rangle & K_{i j}=\left\langle k_{i j}\right\rangle .
\end{array}
$$

A detailed study of the features of the various coefficients can be found in [4] and [8]. It appears that the material presents a biphasic macroscopic behaviour, with a hereditary dynamic Darcy law ( $K$ depends on $\omega$ ). Futhermore, it can be shown that two kinds of compressional waves exist in these media, the second wave being diffusive (consolidation) in the low frequency range, and propagative in the high frequency range [11].

Remark. When the frequency vanishes, the inertial terms in set (11) become negligible, and the dynamic permeability $\mathbf{K}$ tends to the classical intrinsic permeability $\mathbf{k}$ [with $|\mathbf{k}|=O\left(l^{2}\right)$ ].

2.3.2 Elastic macroscopic behaviour.

$$
R T_{L}=O\left(\varepsilon^{-1}\right)
$$

We here study the case $T_{\mathrm{s} L}=O(1), T_{\mathrm{f} L}=O(1), U=O(1), R T_{L}=O\left(\varepsilon^{-1}\right)$ which corresponds to a contrast between viscous and elastic coefficients such as: $\mu \omega /|\mathbf{a}|=O(\varepsilon)$. The equations describing the medium are (1)-(7), but taking into account the new value of $R T_{L}$, equation (5) must be now formally modified to:

$$
\operatorname{grad}(P)+\varepsilon \cdot \mu \cdot \Delta(\mathbf{V})=\rho_{\mathrm{f}} \cdot i \omega \mathbf{V}
$$


Zero order for the solid displacement and the pressure: The problem concerning $\mathbf{U}_{\mathrm{s}}^{0}$ is the same as (8). Consequently we again obtain: $\mathbf{U}_{\mathrm{s}}^{0}(x, y)=\mathbf{U}^{0}(x)$.

About the pressure and the fluid displacement, equations $\left(5^{* *}\right)$ and (4) at $\varepsilon^{-1}$ order, with (6) at zero order give the following differential system:

$$
\mid \begin{array}{ll}
P^{1}(x, y) \text { and } \mathbf{U}_{\mathrm{f}}^{0}(x, y) & \Omega \text {-periodic in } y . \\
\operatorname{grad}_{y}\left(P^{0}\right)+i \omega \mu \cdot \Delta_{y}\left(\mathbf{U}_{\mathrm{f}}^{0}\right)=0 & \text { in } \Omega_{\mathrm{f}} \\
\operatorname{div}_{y}\left(\mathbf{U}_{\mathrm{f}}^{0}\right)=0 & \text { in } \Omega_{\mathrm{f}} \\
\mathbf{U}_{\mathrm{f}}^{0}=\mathbf{U}_{\mathrm{s}}^{0} & \text { on } \Gamma
\end{array}
$$

This system of differential equations is homogeneous with regard to the variable $y$, and the obvious solution is: $\mathbf{U}_{\mathrm{f}}^{0}(x, y)=\mathbf{U}_{\mathrm{s}}^{0}(x, y)=\mathbf{U}^{0}(x), P^{0}(x, y)=P^{0}(x)$.

First order for the solid displacement

From the above results we deduce that equations (2) at $\varepsilon^{-1}$ order and equation (7) at zero order are identical with the one treated in Section 2.3.1. Therefore the solution is the same:

$$
U_{\mathrm{s} i}^{1}=u_{\mathrm{si}}^{1}(x)+\xi_{i j k} \cdot e_{x j k}\left(\mathbf{U}_{\mathrm{s}}^{0}\right)+\eta_{i} \cdot P^{0} .
$$

\section{Compatibility equations}

Concerning the momentum balance, the processing is exactly the same as that used in the previous case. Thus we again obtain, with the same notations:

$$
\operatorname{div}_{x}\left\langle\sigma^{0}\right\rangle=-(1-n) \cdot \rho_{\mathrm{s}} \cdot \omega^{2} \mathbf{U}_{\mathrm{s}}^{0}-n \cdot \rho_{\mathrm{f}} \cdot \omega^{2}\left\langle\mathbf{U}_{\mathrm{f}}^{0}\right\rangle=-\langle\rho\rangle \cdot \omega^{2} \mathbf{U}^{0}
$$

where $\langle\rho\rangle=(1-n) \cdot \rho_{\mathrm{s}}-n \cdot \rho_{\mathrm{f}}$ is the bulk density.

With regards to the volume balance, we now obtain:

$$
n \cdot \operatorname{div}_{x}\left(\mathbf{U}^{0}\right)=\int_{\Omega_{\mathrm{s}}} \operatorname{div}_{y}\left(\mathbf{U}_{\mathrm{s}}^{1}\right) \cdot \mathrm{d} \Omega
$$

\section{Macroscopic equivalent behaviour}

Replacing $\mathbf{U}_{\mathrm{s}}^{1}$ by its expression, yields the following set of equations valid at the first order.

with

$$
\mid \begin{aligned}
& \langle\sigma\rangle=\mathbf{C} \cdot \mathbf{e}(\mathbf{U})+\boldsymbol{\alpha} \cdot P \\
& \operatorname{div}\langle\boldsymbol{\sigma}\rangle=-\langle\rho\rangle \cdot \omega^{2} \mathbf{U} \\
& 0=-\alpha \cdot \mathbf{e}(\mathbf{U})+\beta \cdot P
\end{aligned}
$$

$$
\begin{aligned}
& C_{i j k l}=\left\langle a_{i j k l}+a_{i j m n} \cdot e_{y m n}\left(\xi_{k l}\right)\right\rangle \quad \beta=\left\langle\operatorname{div}_{y}(\boldsymbol{\eta})\right\rangle \\
& \alpha_{i j}=n \cdot \delta_{i j}+\left\langle a_{i j m n} \cdot e_{y m n}(\boldsymbol{\eta})\right\rangle .
\end{aligned}
$$

This system can be rewritten as:

with

$$
\mid \begin{aligned}
& \langle\boldsymbol{\sigma}\rangle=\mathbf{C}^{*} \cdot \mathbf{e}(\mathbf{U}) \\
& \operatorname{div}\langle\boldsymbol{\sigma}\rangle=-\langle\rho\rangle \cdot \omega^{2} \mathbf{U}
\end{aligned}
$$

$$
C_{i j k l}^{*}=\left\langle a_{i j k l}+a_{i j m n} \cdot e_{y m n}\left(\xi_{k l}\right)\right\rangle+\beta^{-1} \cdot \alpha_{i j} \cdot \alpha_{k l} .
$$

Therefore the homogenized medium has an elastic monophasic behaviour $\left(\mathbf{C}^{*}\right.$ is a real positive tensor). It must be pointed out that the viscosity of the fluid does not influence the macroscopic level: the viscosity is high enough to suppress the relative displacement between the fluid and the solid, but too weak to trasmit shear stresses on the macroscopic scale.

2.3.3 Viscoelastic macroscopic behaviour

$$
R T_{L}=O(1)
$$

At last, let us consider the case where $T_{\mathrm{s} L}=O(1), T_{\mathrm{f} L}=O(1), U=O(1), R T_{L}=O(1)$. These conditions are satisfied as soon as: $\mu \omega /|\mathbf{a}|=O(1)$.

As there is no contrast between the mechanical parameters of the constituants, we do not have to renormalize equation (5). 
Zero order for the solid and fluid displacements

The problem to be solved at the first order is:

$$
\mid \begin{array}{ll}
\mathbf{U}_{\mathrm{s}}^{0}(x, y) \text { and } \mathbf{U}_{\mathrm{f}}^{0}(x, y) & \Omega \text {-periodic in } y . \\
\operatorname{div}_{y}\left(\mathbf{a} \cdot\left(\mathbf{e}_{y}\left(\mathbf{U}_{\mathrm{s}}^{0}\right)\right)\right)=0 & \text { in } \Omega_{\mathrm{s}} \\
\left(\mathbf{a} \cdot \mathbf{e}_{y}\left(\mathbf{U}_{\mathrm{s}}^{0}\right)-2 i \omega \mu \cdot \mathbf{e}_{y}\left(\mathbf{U}_{\mathrm{f}}^{0}\right)\right) \cdot \mathbf{N}=0 & \text { on } \Gamma \\
2 i \omega \mu \cdot \mathbf{\Delta}_{y}\left(\mathbf{U}_{\mathrm{f}}^{0}\right)=0 & \text { in } \boldsymbol{\Omega}_{\mathrm{f}} \\
\operatorname{div}_{y}\left(\mathbf{U}_{\mathrm{f}}^{0}\right)=0 & \text { in } \Omega_{\mathrm{f}} \\
\mathbf{U}_{\mathrm{f}}^{0}=\mathbf{U}_{\mathrm{s}}^{0} & \text { on } \Gamma
\end{array}
$$

Once more this is an homogeneous problem in $y$ variable, the obvious solution of which is

$$
\mathbf{U}_{\mathrm{f}}^{0}(x, y)=\mathbf{U}_{\mathrm{s}}^{0}(x, y)=\mathbf{U}^{0}(x)
$$

First order for the solid displacements, zero order for the pressure

The previous equations taken at the next order lead to the following boundary value problem:

$$
\mid \begin{array}{ll}
\mathbf{U}_{\mathbf{s}}^{1}(x, y), \mathbf{U}_{\mathbf{f}}^{1}(x, y) \quad \text { and } P^{0}(x, y) & \Omega \text {-periodic in } y . \\
\operatorname{div}_{y}\left(\mathbf{a} \cdot\left(\mathbf{e}_{y}\left(\mathbf{U}_{\mathrm{s}}^{1}\right)+\mathbf{e}_{x}\left(\mathbf{U}^{0}\right)\right)\right)=0 & \text { in } \Omega_{\mathrm{s}} \\
\left(\mathbf{a} \cdot\left(\mathbf{e}_{y}\left(\mathbf{U}_{\mathrm{s}}^{1}\right)+\mathbf{e}_{x}\left(\mathbf{U}^{0}\right)\right)\right) \cdot \mathbf{N}=\left(P^{0} \cdot \mathbf{I}+2 i \omega \mu \cdot\left(\mathbf{e}_{y}\left(\mathbf{U}_{\mathrm{f}}^{1}\right)+\mathbf{e}_{x}\left(\mathbf{U}^{0}\right)\right)\right) \cdot \mathbf{N} & \text { on } \Gamma \\
\operatorname{grad}_{y}\left(P^{0}\right)+2 i \omega \mu \cdot \mathbf{\Delta}_{y}\left(\mathbf{U}_{\mathrm{f}}^{1}\right)=0 & \text { in } \Omega_{\mathrm{f}} \\
\operatorname{div}_{y}\left(\mathbf{U}_{\mathrm{f}}^{1}\right)+\operatorname{div}_{x}\left(\mathbf{U}^{0}\right)=0 & \text { in } \Omega_{\mathrm{f}} \\
\mathbf{U}_{\mathrm{f}}^{1}=\mathbf{U}_{\mathrm{s}}^{1} & \text { on } \Gamma
\end{array}
$$

We again obtain a well posed linear problem, and the solution depends linearly on the macroscopic assigned strain tensor $e_{x}\left(U^{0}\right)$. Thus, putting down: $\mathbf{U}^{1}=\mathbf{U}_{\mathrm{s}}^{1}$ in $\Omega_{\mathrm{s}},=\mathbf{U}_{\mathrm{f}}^{1}$ in $\Omega_{\mathrm{f}}$, we have:

$$
U_{i}^{1}=u_{i}^{1}(x)+\chi_{i j k} \cdot e_{x j k}\left(\mathbf{U}^{0}\right) \quad P^{0}=\tau_{j k} \cdot e_{x j k}\left(\mathbf{U}^{0}\right) .
$$

Note that $\mathbf{U}^{1}$ and $P^{0}$ have a complex value.

Compatibility equation

In the present case, only one compatibility equation remains: the momentum balance. The process is again identical to the one used in the Section 2.3.1, and as the fluid and solid displacements are the same:

$$
\operatorname{div}_{x}\left\langle\sigma^{0}\right\rangle=-\langle\rho\rangle \cdot \omega^{2} \mathbf{U}^{0}
$$

Macroscopic behaviour of the equivalent continuum

The behaviour is given by the following equations:

$$
\begin{gathered}
\langle\boldsymbol{\sigma}\rangle=\mathbf{C}^{* *} \cdot \mathbf{e}(\mathbf{U}) \\
\operatorname{div}\langle\boldsymbol{\sigma}\rangle=-\langle\rho\rangle \cdot \omega^{2} \mathbf{U}
\end{gathered}
$$

with: $C_{i j k l}^{* *}=(1-n) \cdot a_{i j k l}+n \cdot 2 \cdot i \omega \mu \delta_{i j} \cdot \delta_{k l}$

$$
+\int_{\Omega_{\mathrm{s}}} a_{i j m n} \cdot e_{y m n}\left(\chi_{k l}\right) \cdot \mathrm{d} \Omega+\int_{\Omega_{i}}\left(2 \cdot i \omega \mu \cdot e_{y i j}\left(\chi_{k l}\right)+\tau_{i j} \cdot \delta_{k l}\right) \mathrm{d} \Omega .
$$

It must be noted that the equivalent medium is monophasic, and since $\mathbf{C}^{* *}$ is a complex tensor, the material is macroscopically viscoelastic.

\subsubsection{Conclusions}

Using homogenization, we have pointed out three distinct types of macroscopic behaviour, i.e. biphasic with a dynamic Darcy law, monophasic elastic or viscoelastic, according to the magnitude of the contrast $\mu \omega /|\mathbf{a}|$, measured by the small parameter $\varepsilon$. One ought to remember that these types of behaviour are given at the first order only, and that $\varepsilon$ corresponds to the accuracy of the description obtained by the homogenization process. 


\subsection{Homogenization of a porous medium saturated by a viscoelastic fluid}

Let us come back to the study developed in preceding Section 2.3, but assuming now that the saturating fluid is an incompressible, viscoelastic linear one. Under this new hypothesis, the deviatoric stress in the fluid involves a viscous part (as for a Newtonian fluid), and also an elastic part. The constitutive law of this fluid undergoing harmonic excitations can be expressed as follows:

$$
\sigma_{\mathrm{f}}=P \cdot \mathbf{1}+2 \cdot M(\omega) \cdot \mathbf{e}\left(\mathbf{U}_{\mathrm{f}}\right) .
$$

$M(\omega)$ is the complex modulus depending on the frequency: $M(\omega)=M_{r}+i \cdot M_{i}, M_{r}$ and $M_{i} / \omega$ correspond to the apparent elastic modulus and the viscosity at the pulsation $\omega$, respectively.

In comparison with the previous case, only the constitutive law of the fluid is modified in the microscopic description. So the expressions of the dimensionless numbers remain the same but for the transient Reynolds number $R T^{\prime}$, which is now written as the ratio between inertial forces and deviatoric stresses. Using $l$ as reference length, we have:

$$
R T_{1}^{\prime}=\rho_{f} \cdot|\partial \mathbf{V} / \partial t| /|M \cdot \Delta(\mathbf{V})| \approx \rho_{\mathrm{f}} \cdot \omega \cdot l^{2} /|M| .
$$

Later on, for the same reason as in the previous section, we will use $R T_{L}^{\prime}=\varepsilon^{-2} \cdot R T_{l}^{\prime}$. Substituting $i \omega \mu$ by $M(\omega)$, in the study presented in 2.3 , the homogenization can be performed in exactly the same way. Thus we obtain the three different models according to the contrast between the norm of the complex modulus $|M(\omega)|$ and that of the elastic tensor $|a|$, i.e. to the magnitude of $R T_{L}^{\prime}$.

2.4.1 Macroscopic biphasic behaviour. $R T_{L}^{\prime}=O\left(\varepsilon^{-2}\right)$ or $|M(\omega)| /|\mathbf{a}|=O\left(\varepsilon^{2}\right)$

Let us first assume that $M_{r}$ and $M_{i}$ are of the same order of magnitude. In the same manner, step by step, as in Section 2.3.1, it can be seen that the only modification appears in system (12). For this reason all the previous results are still valid, except the generalized dynamic Darcy law, and we only have to introduce particular solutions $\mathbf{W}^{\prime 0}\left(=\mathbf{k}_{i}^{\prime} / M\right)$ similar to solutions $\mathbf{W}^{0}\left(=\mathbf{k}_{i} / i \omega \mu\right)$, such as:

$$
\begin{aligned}
& \mid \begin{array}{ll}
P^{1}(x, y) \text { and } \mathbf{W}^{\prime 0}(x, y) & \Omega \text {-periodic in } y . \\
\operatorname{grad}_{y}\left(P^{1}\right)+M \cdot \mathbf{\Delta}_{y}\left(\mathbf{W}^{\prime 0}\right)+\rho_{f} \cdot \omega^{2} \cdot \mathbf{W}^{\prime 0}=-\operatorname{grad}_{x}\left(P^{0}\right)-\rho_{f} \cdot \omega^{2} \mathbf{U}_{\mathrm{s}}^{0} & \text { in } \Omega_{\mathrm{f}} \\
\operatorname{div}_{y}\left(\mathbf{W}^{\prime 0}\right)=0 & \text { in } \Omega_{\mathrm{f}} \\
\mathbf{W}^{\prime 0}=0 & \text { on } \Gamma
\end{array} \\
& \text { when }\left(\operatorname{grad}_{x}\left(P^{0}\right)+\rho_{f} \cdot \omega^{2} U_{s}^{0}\right)_{j}=\delta_{i j}
\end{aligned}
$$

Therefore the macroscopic behaviour is given by:

with:

$$
\mid \begin{aligned}
& \langle\boldsymbol{\sigma}\rangle=\mathbf{C} \cdot \mathbf{e}\left(\mathbf{U}_{\mathrm{s}}\right)+\boldsymbol{\alpha} \cdot P \\
& \operatorname{div}\langle\boldsymbol{\sigma}\rangle=-(1-n) \cdot \rho_{\mathrm{s}} \cdot \omega^{2} \mathbf{U}_{\mathrm{s}}-n \cdot \rho_{f} \cdot \omega^{2}\left\langle\mathbf{U}_{\mathrm{f}}\right\rangle \\
& \left\langle\mathbf{U}_{\mathrm{f}}\right\rangle-n \cdot \mathbf{U}_{\mathrm{s}}=\left(\mathbf{K}^{\prime} / M\right) \cdot\left(\operatorname{grad}(P)+\rho_{f} \cdot \omega^{2} \mathbf{U}_{\mathrm{s}}\right) \\
& \operatorname{div}\left(\left\langle\mathbf{U}_{\mathrm{f}}\right\rangle-n \cdot \mathbf{U}_{\mathrm{s}}\right)=-\boldsymbol{\alpha} \cdot \mathbf{e}\left(\mathbf{U}_{\mathrm{s}}\right)+\beta \cdot P
\end{aligned}
$$

$$
\begin{array}{ll}
C_{i j k l}=\left\langle a_{i j k l}+a_{i j m n} \cdot e_{y m n}\left(\xi_{k l}\right)\right\rangle & \beta=\left\langle\operatorname{div}_{y}(\boldsymbol{\eta})\right\rangle \\
\alpha_{i j}=n \cdot \delta_{i j}+\left\langle a_{i j m n} \cdot e_{y m n}(\boldsymbol{\eta})\right\rangle & K_{i j}^{\prime}=\left\langle k_{i j}^{\prime}\right\rangle
\end{array}
$$

Remarks

- As in the case of a viscous Newtonian fluid, set (16) describes a biphasic behaviour. Equation (16c), which gives the relative fluid displacement, corresponds to a Darcy law (with a filtration coefficient depending on the frequency). Consequently there are two kinds of compressional waves (with dispersion phenomena), in this medium.

-The tensors $\mathbf{C}, \boldsymbol{\alpha}$, and the coefficient $\beta$ are identical to those defined in Section 2.3.1. On the contrary, $\mathbf{K}^{\prime}=\left\langle\mathbf{k}^{\prime}\right\rangle$ is distinct from $\mathbf{K}$. In fact equation (16c) involves two memory effects: one due to the dynamic coupling between the fluid and the solid, and the other one due to the own memory of the fluid.

-If the frequency is low enough to neglect the inertial effects, the sets of equations (12) and (15) are similar and their solutions $\mathbf{W}^{0}$ and $\mathbf{W}^{\prime 0}$ become proportional, in the ratio 
$M / i \omega \mu$. Therefore, in this frequency range, $\mathbf{K}^{\prime}=\mathbf{K}=\mathbf{k}$ : the intrinsic permeabilities are equal but, of course, the corresponding Darcy laws are different $[\mu$ is real, $M(\omega) / i \omega$ is complex].

-If we assume that the elastic term $M_{r}$ is negligible [say $O(\varepsilon)$ ] with regard to the viscous term $M_{i}$, we obtain exactly the same formulation as in the case of a viscous fluid having a viscosity of $\mu=M_{i} / \omega$.

-On the contrary, if we suppose that $M_{i}$ is negligible [say $O(\varepsilon)$ ] with regard to $M_{r}$, the fluid behaves as an incompressible elastic material. This unclassical case has already been treated by Auriault and Bonnet in [6]. The study shows the existence of cut off frequencies associated with the resonance modes of the fluid into the connected pores.

-In the case $M_{r} / M_{i}=O(1)$, the effects of dispersion due to the resonance modes are damped, more especially if the apparent viscosity becomes important.

2.4.2 Elastic macroscopic behaviour. $R T_{L}^{\prime}=O\left(\varepsilon^{-1}\right)$ or $|M(\omega)| /|\mathbf{a}|=O(\varepsilon)$

In this case, as the fluid only intervenes in the problems by its pressure, the process of homogenization is absolutely the same as that developed in 1.3.2. Therefore we have:

$$
\begin{aligned}
\langle\boldsymbol{\sigma}\rangle & =\mathbf{C}^{*} \cdot \mathbf{e}(\mathbf{U}) \\
\operatorname{div}\langle\boldsymbol{\sigma}\rangle & =-\langle\rho\rangle \cdot \omega^{2} \mathbf{U} .
\end{aligned}
$$

The expression of the tensor $\mathbf{C}^{*}$ is the same as the one given in 2.3.2.

\section{Remark}

At the first order, for a given microstructure, the macroscopic description is independant of the rheology of the incompressible fluid.

2.4.3 Viscoelastic macroscopic behaviour. $R T_{L}^{\prime}=O(1)$ or $|M(\omega)| /|\mathbf{a}|=O(1)$

Let us first consider the case where: $M_{r} / M_{i}=O(1)$. If we come back to the differential system (13), we can see that only the boundary condition concerning the stresses is modified. Nevertheless the problem remains homogeneous, and we again get: $\mathbf{U}_{\mathrm{f}}^{0}(x, y)=\mathbf{U}_{\mathrm{s}}^{0}(x, y)=$ $\mathbf{U}^{0}(x)$. In the second problem (14), we only have to replace $i \omega \mu$ by $M(\omega)$. And in the same way as previously, we deduce that:

with

$$
\mid \begin{aligned}
& \langle\boldsymbol{\sigma}\rangle=\mathbf{C}^{* *} \cdot \mathbf{e}(\mathbf{U}) \\
& \operatorname{div}\langle\boldsymbol{\sigma}\rangle=-\langle\rho\rangle \cdot \omega^{2} \mathbf{U}
\end{aligned}
$$

$$
\begin{aligned}
C_{i j k l}^{\prime * *}= & (1-n) \cdot a_{i j k l}+n \cdot 2 \cdot M(\omega) \cdot \delta_{i j} \cdot \delta_{k l} \\
& +\int_{\Omega_{\mathrm{s}}} a_{i j m n} \cdot e_{y m n}\left(\chi_{k l}^{\prime}\right) \cdot \mathrm{d} \Omega+\int_{\Omega_{\mathrm{f}}}\left(2 \cdot M(\omega) \cdot e_{y i j}\left(\chi_{k l}^{\prime}\right)+\tau_{i j}^{\prime} \cdot \delta_{k l}\right) \mathrm{d} \Omega .
\end{aligned}
$$

Consequently we obtain a viscoelastic behaviour, different from that corresponding to a Newtonian saturating fluid [since $\chi_{j k}^{\prime}$ and $\boldsymbol{\tau}_{j k}^{\prime}$, solutions of (14) where $i \omega \mu$ is replaced by $M(\omega)$, are complex and distinct from $\boldsymbol{\chi}_{j k}$ and $\boldsymbol{\tau}_{j k}$ ].

Remarks

-When $M_{r} / M_{i}=O(\varepsilon)$, we come back to the case of a Newtonian fluid with a viscosity $\mu=M_{i} / \omega$.

-On the contrary, if $M_{r} / M_{i}=O\left(\varepsilon^{-1}\right)$, set (14) which allows the determination of $\chi^{\prime}$ and $\tau^{\prime}$, becomes real. Consequently this case leads to a new macroscopical elastic behaviour, distinct from that obtained in Section 2.4.2.

\section{STUDY OF A UNIDIRECTIONAL MODEL}

In this section we apply the previous results to a very simple (1D) microscopic structure, which allows us to point out the three kinds of behaviour in an analytical way.

Let us consdier a stratified medium constituted by an alternance of homogeneous solid layers and viscoelastic layers, respectively with thickness of $(1-n) \cdot h$, and $n \cdot h$ (see Fig. 2). The two components are supposed to be isotropic. $\lambda$ and $\mu$ are the Lame constants of the elastic part. For this very particular geometry, the volumes $\Omega_{\mathrm{s}}$ and $\Omega_{\mathrm{f}}$ are connected only in the directions 


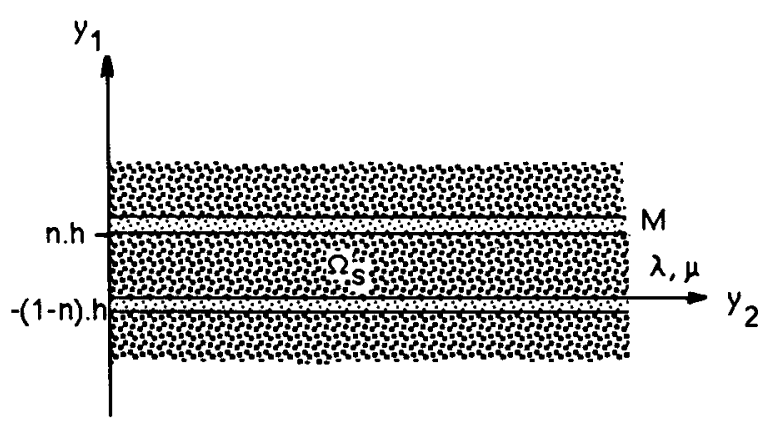

Fig. 2. Geometry of the stratified medium.

$y_{2}$ and $y_{3}$. The medium presents a cylindrical symmetry around the $y_{1}$ axis. Moreover, the period is undefined for the $y_{2}$ and $y_{3}$ directions, consequently, the local fields depend only on the variable $y_{1}$.

Let us compute the various coefficients introduced by each of the behaviour laws.

\subsection{Biphasic behaviour}

This behaviour is characterized by the four tensors $\mathbf{C}, \boldsymbol{\alpha}, \beta, \mathbf{K}^{\prime}$

3.1.1 Elastic coefficients: computation of $\mathbf{C}, \boldsymbol{\alpha}, \beta$. We have seen that:

$$
C_{i j k l}=\left\langle a_{i j k l}+a_{i j m n} \cdot e_{y m n}\left(\xi_{k l}\right)\right\rangle
$$

where $\xi_{j k}$ is the vector solution of the particular problem:

$$
\mid \begin{array}{ll}
\mathbf{U}_{\mathrm{s}}^{1}(x, y) & \mathbf{\Omega} \text {-periodic in } y \\
\operatorname{div}_{y}\left(\mathbf{a} \cdot\left(\mathbf{e}_{y}\left(\mathbf{U}_{\mathrm{s}}^{1}\right)+\mathbf{e}_{x}\left(\mathbf{U}_{\mathrm{s}}^{0}\right)\right)\right)=0 & \text { in } \boldsymbol{\Omega}_{\mathrm{s}} \\
\left(\mathbf{a} \cdot\left(\mathbf{e}_{y}\left(\mathbf{U}_{\mathrm{s}}^{1}\right)+\mathbf{e}_{x}\left(\mathbf{U}_{\mathrm{s}}^{0}\right)\right) \cdot \mathbf{N}=P^{0} \cdot \mathbf{N}\right. & \text { on } \Gamma \\
\text { with: } P^{0}=0, e_{x l m}\left(\mathbf{U}_{\mathrm{s}}^{0}\right)=\left(\delta_{j l} \cdot \delta_{k m}+\delta_{j m} \cdot \delta_{k l}\right) / 2
\end{array}
$$

Taking into account the isotropy of the two materials, the homogeneity of the layers and the fact that $\mathbf{U}_{\mathrm{s}}^{1}$ only depends on $y_{1}$, the set (17) can be rewritten as follows:

$$
(\lambda+2 \cdot \mu) d^{2} U_{\mathrm{s} 1}^{1} / d y_{1}^{2}=2 \cdot \mu d^{2} U_{\mathrm{s} 2}^{1} / d y_{1}^{2}=2 \cdot \mu d^{2} U_{\mathrm{s} 3}^{1} / d y_{1}^{2}=0
$$

for $y_{1}=0$ :

$$
\begin{aligned}
& 0=\left[(\lambda+2 \cdot \mu) d U_{\mathrm{s} 1}^{1} / d y_{1}+\left(2 \cdot \mu e_{11}+\lambda \cdot \theta\right)\right]_{y_{1}=0} \\
& 0=\left[2 \cdot \mu d U_{\mathrm{s} 2}^{1} / d y_{1}+2 \cdot \mu e_{12}\right]_{y_{1}=0} \\
& 0=\left[2 \cdot \mu d U_{\mathrm{s} 3}^{1} / d y_{1}+2 \cdot \mu e_{13}\right]_{y_{1}=0}
\end{aligned}
$$

where $e_{i j}$ and $\theta$ stands for $e_{x i j}\left(\mathbf{U}_{s}^{0}\right)$ and $\operatorname{div}_{x}\left(\mathbf{U}_{s}^{0}\right)$, respectively. We easily obtain:

$$
\begin{aligned}
& U_{\mathrm{s} 1}^{1}=-y_{1}\left(2 \mu e_{11}+\lambda \cdot \theta\right) \cdot(\lambda+2 \mu)^{-1}+u_{\mathrm{s} 1}^{1}(x)=\xi_{1 j k} \cdot e_{j k}+u_{\mathrm{s} 1}^{1}(x) \\
& U_{\mathrm{s} 2}^{1}-2 \cdot e_{12} \cdot y_{1}+u_{\mathrm{s} 2}^{1}(x)=\xi_{2 j k} \cdot e_{j k}+u_{\mathrm{s} 2}^{1}(x) \\
& U_{\mathrm{s} 3}^{1}=-2 \cdot e_{13} \cdot y_{1}+u_{\mathrm{s} 3}^{1}(x)=\xi_{3 j k} \cdot e_{j k}+u_{\mathrm{s} 3}^{1}(x) .
\end{aligned}
$$

We then calculate the vectors $\xi_{j k}$ and determine the terms $\left\langle a_{i j m n} \cdot e_{y m n}\left(\xi_{k l}\right)\right\rangle$. After computation, writing the macroscopic elastic law on the matrical form, $\sigma=\mathbf{C} \cdot \varepsilon$, with:

$$
\boldsymbol{\sigma}=\left(\sigma_{11}, \sigma_{22}, \sigma_{33}, \sigma_{23}, \sigma_{13}, \sigma_{12}\right), \quad \varepsilon={ }^{t}\left(e_{11}, e_{22}, e_{33}, e_{23}, e_{13}, e_{12}\right)
$$


the symmetrical matrix $C$ of the elastic coefficients is equal to:

$$
\mathbf{C}=\left[\begin{array}{ccccc}
0 & 0 & 0 & & \\
0 & C_{22} & C_{23} & & \\
0 & C_{23} & C_{22} & & \\
& & & C_{44} & \\
& & & & 0 \\
& & & &
\end{array}\right] \begin{aligned}
& C_{22}=(1-n) \cdot\left[(\lambda+2 \cdot \mu)^{2}-\lambda^{2}\right](\lambda+2 \cdot \mu)^{-1} \\
& C_{23}=(1-n) \cdot 2 \cdot \mu \cdot \lambda \cdot(\lambda+2 \cdot \mu)^{-1} \\
& C_{44}=(1-n) \cdot \mu .
\end{aligned}
$$

The tensor $\alpha$ and the coefficient $\beta$ are given by:

$$
\beta=\left\langle\operatorname{div}_{y}(\boldsymbol{\eta})\right\rangle \quad \alpha_{i j}=n \cdot \delta_{i j}+\left\langle a_{i j m n} \cdot e_{y m n}(\boldsymbol{\eta})\right\rangle .
$$

The vector $\eta$ is the particular solution of (17) with: $\mathbf{e}_{x}\left(\mathbf{U}^{0}\right)=0, P^{0}=1$

$$
(\lambda+2 \cdot \mu) d^{2} \eta_{1} / d y_{1}^{2}=2 \cdot \mu d^{2} \eta_{2} / d y_{1}^{2}=2 \cdot \mu \cdot d^{2} \eta_{3} / d y_{1}^{2}=0
$$

for $y_{1}=0$

Consequently:

$$
\left[(\lambda+2 \cdot \mu) d \eta_{1} / d y_{1}-1\right]_{y_{1}=0}=0 .
$$

So, we easily obtain:

$$
\begin{aligned}
& \eta_{1}=y_{1} \cdot(\lambda+2 \cdot \mu)^{-1}+u_{\mathrm{s} 1}^{1}(x) \\
& \eta_{2}=u_{\mathrm{s} 2}^{1}(x) \\
& \eta_{3}=u_{\mathrm{s} 3}^{1}(x)
\end{aligned}
$$

$$
\begin{aligned}
\alpha_{i j} & \left.=\delta_{i j} \cdot\left((1-n) \cdot\left(\lambda+2 \cdot \mu \cdot \delta_{1 i}\right) \cdot(\lambda+2 \cdot \mu)^{-1}+n\right) \quad \text { (without summation on } i\right) \\
\beta & =(1-n) \cdot(\lambda+2 \cdot \mu)^{-1}
\end{aligned}
$$

3.1.2 Permeability tensor $\mathbf{K}^{\prime}(w)$. $\mathbf{K}^{\prime}(\omega)$ is defined by $K_{i j}^{\prime}=\left\langle k_{i j}^{\prime}\right\rangle$, where $\mathbf{k}_{i}^{\prime} / M$ is the vector $\mathbf{W}^{\prime 0}$ solution of the problem:

$$
\begin{aligned}
& \mid \begin{array}{ll}
P^{1}(x, y) \text { and } \mathbf{W}^{\prime 0}(x, y) & \Omega \text {-periodic in } y . \\
\operatorname{grad}_{y}\left(P^{1}\right)+M \cdot \Delta_{y}\left(\mathbf{W}^{\prime 0}\right)+\rho_{\mathrm{f}} \cdot \omega^{2} \mathbf{W}^{\prime 0}=-\operatorname{grad}_{x}\left(P^{0}\right)-\rho_{\mathrm{f}} \cdot \omega^{2} \mathbf{U}_{\mathrm{s}}^{0} & \text { in } \Omega_{\mathrm{f}} \\
\operatorname{div}_{y}\left(\mathbf{W}^{\prime 0}\right)=0 & \text { in } \Omega_{\mathrm{f}} \\
\mathbf{W}^{\prime 0}=0 & \text { on } \Gamma
\end{array} \\
& \text { with }\left(\operatorname{grad}_{x}\left(P^{0}\right)+\rho_{\mathrm{f}} \cdot \omega^{2} \mathbf{U}_{\mathrm{s}}^{0}\right)_{j}=\delta_{i j}
\end{aligned}
$$

As $\mathbf{W}^{\prime 0}$ only depends on the variable $y_{1}$, the incompressibility together with the adherence condition at the interface, implies $\mathbf{W}_{1}^{\prime 0}=0$.

Thus we have to solve:

$$
\mid \begin{aligned}
& P_{, y 1}^{1}=-\delta_{i 1} \\
& M \cdot d^{2} W_{2}^{\prime 0} / d y_{1}^{2}+\rho_{f} \cdot \omega^{2} W_{2}^{\prime 0}=-\delta_{i 2} \\
& M \cdot d^{2} W_{3}^{\prime 0} / d y_{1}^{2}+\rho_{f} \cdot \omega^{2} W_{3}^{\prime 0}=-\delta_{i 3}
\end{aligned}
$$

-If $i=1$ (Macroscopic pressure gradient perpendicular to the layers), there is no flow: $\mathbf{W}^{\prime 0}=0$ and thus $k_{1}^{\prime}=0$.

-If $i=2$ (Macroscopic pressure gradient parallel to the $y_{2}$ axis, i.e., to the layers)

$$
\begin{aligned}
& W_{1}^{\prime 0}=0=W_{3}^{\prime 0}, \\
& W_{2}^{\prime 0}=\left(\rho_{\mathrm{f}} \cdot \omega^{2}\right)^{-1} \cdot\left(-1+A \cdot \exp \left[i \omega\left(\rho_{\mathrm{f}} / M\right)^{1 / 2} \cdot y_{1}\right]+B \cdot \exp \left[-i \omega\left(\rho_{\mathrm{f}} / M\right)^{1 / 2} \cdot y_{1}\right]\right. \\
& \text { (with } \left.R_{e}\left[(z)^{1 / 2}\right]>0\right) .
\end{aligned}
$$

The boundary conditions: $W_{2}^{\prime 0}=0$ for $y_{1}=0$ and for $y_{1}=n \cdot h$, allow the calculation of $A$ and $B$ :

$$
A=\left\{1+\exp \left[i \omega \cdot n h\left(\rho_{\mathrm{f}} / M\right)^{1 / 2}\right]\right\}^{-1} \quad B=\left\{1+\exp \left[-i \omega \cdot n h\left(\rho_{\mathrm{f}} / M\right)^{1 / 2}\right]\right\}^{-1}
$$


Then, after integration over the period, and multiplying by $M$, is obtained:

$$
\left\langle k_{22}^{\prime}\right\rangle=\left(n \cdot M / \rho_{\mathrm{f}} \cdot \omega^{2}\right)\left[-1+2 \cdot\left[i\left(\rho_{\mathrm{f}} / M\right)^{1 / 2} \omega \cdot n h / 2\right]^{-1} \cdot \operatorname{tg}\left[\left(\rho_{\mathrm{f}} / M\right)^{1 / 2} \omega \cdot n h / 2\right] .\right.
$$

And introducing the complex adimensional pulsation $\omega^{*}$ defined by: $\omega^{*}=\omega \cdot\left(\rho_{\mathrm{f}} / M\right)^{1 / 2} n h / 2$ this expression becomes:

$$
\left\langle k_{22}^{\prime}\right\rangle=K^{\prime}=M \cdot\left(n / \rho_{\mathrm{f}} \cdot \omega^{2}\right)\left(-1+\operatorname{tg}\left(\omega^{*}\right) / i \omega^{*}\right)
$$

$-j=3$ leads to a similar result.

Finally, the permeability tensor takes the form:

$$
K^{\prime}=\left[\begin{array}{ccc}
0 & 0 & 0 \\
0 & K^{\prime} & 0 \\
0 & 0 & K^{\prime}
\end{array}\right]
$$

3.1.3 Summary. The biphasic behaviour of the stratified medium is defined by:

-The stress tensor:

$$
\langle\sigma\rangle=\left[\begin{array}{ccc}
P & 0 & 0 \\
0 & \sigma_{22} & \sigma_{23} \\
0 & \sigma_{23} & \sigma_{33}
\end{array}\right] \quad \begin{aligned}
& \sigma_{i i}=[(1-n) \lambda /(\lambda+2 \mu)+n] P \\
& +(1-n)\left(\lambda[1-\lambda /(\lambda+2 \mu)]\left(e_{22}+e_{33}\right)+2 \cdot \mu e_{i i}\right) \\
& \text { (without summation on } i) \\
& \sigma_{23}=(1-n) 2 \cdot \mu e_{23}
\end{aligned}
$$

-the filtration law:

$$
\left\langle\mathbf{U}_{\mathrm{f}}\right\rangle-n \cdot \mathbf{U}_{\mathrm{s}}=\left(\mathbf{K}^{\prime} / M\right)\left[\operatorname{grad}(P)+\rho_{\mathrm{f}} \cdot \omega^{2} \mathbf{U}_{\mathrm{s}}\right]
$$

-The momentum balance:

-and the volume balance:

$$
\operatorname{div}\langle\sigma\rangle=-(1-n) \cdot \rho_{\mathrm{s}} \cdot \omega^{2} \mathbf{U}_{\mathrm{s}}-n \cdot \rho_{\mathrm{f}} \cdot \omega^{2}\left\langle\mathbf{U}_{\mathrm{f}}\right\rangle
$$

$$
\operatorname{div}\left(\left\langle\mathbf{U}_{\mathrm{f}}\right\rangle-n \cdot \mathbf{U}_{\mathrm{s}}\right)=-e_{11}-\left(e_{22}+e_{33}\right)[(1-n) \lambda /(\lambda+2 \mu)+n]+P(1-n) /(\lambda+2 \mu) .
$$

\subsection{Elastic behaviour}

As we have seen in Section 2.3.2 the elastic behaviour can be obtained from the biphasic behaviour, by expressing that:

$$
\mathbf{U}_{\mathrm{f}}-\mathbf{U}_{\mathrm{s}}=0, \text { thus } \operatorname{div}_{x}\left(\left\langle\mathbf{U}_{\mathbf{f}}\right\rangle-n \cdot \mathbf{U}_{\mathrm{s}}\right)=0, \text { which leads to: } \mathbf{C}^{*}=\mathbf{C}+\boldsymbol{\alpha} \cdot \boldsymbol{\alpha} \cdot \boldsymbol{\beta}^{-1} \text {. }
$$

By using the same notations as above, the symmetrical matrix of the elastic coefficients is written:

$$
C^{*}=\left[\begin{array}{llllll}
C_{11}^{*} & C_{12}^{*} & C_{12}^{*} & & & \\
& C_{22}^{*} & C_{23}^{*} & & & \\
& & C_{22}^{*} & & & \\
& & & C_{44}^{*} & & \\
& & & & 0 & \\
& & & & & 0
\end{array}\right] \begin{aligned}
& C_{11}^{*}=(\lambda+2 \cdot \mu)(1-n)^{-1} \\
& C_{12}^{*}=(\lambda+2 \cdot n \cdot \mu)(1-n)^{-1} \\
& C_{23}^{*}=\left(\lambda+n^{2} \cdot 2 \cdot \mu\right)(1-n)^{-1} \\
& C_{22}^{*}=(\lambda+2 \cdot \mu)(1-n)^{-1}-n \cdot 4 \cdot \mu \\
& C_{44}^{*}=(1-n) \cdot \mu
\end{aligned}
$$

\subsection{Viscoelastic behaviour}

The viscoelastic tensor $\mathbf{C}^{* *}$ is determined from the particular solutions $\mathbf{U}_{\mathrm{s}}^{1}(x, y)=\chi_{j k}^{\prime}$ and $P^{1}(x, y)=\tau_{j k}^{\prime}$ of the following boundary conditions problem:

$$
\mid \begin{array}{ll}
\mathbf{U}_{\mathrm{s}}^{1}(x, y), \mathbf{U}_{\mathbf{f}}^{1}(x, y) \quad \text { and } P^{0}(x, y) & \mathbf{\Omega} \text {-periodic in } y . \\
\operatorname{div}_{y}\left(\mathbf{a} \cdot\left(\mathbf{e}_{y}\left(\mathbf{U}_{\mathrm{s}}^{1}\right)+\mathbf{e}_{x}\left(\mathbf{U}^{0}\right)\right)\right)=0 & \text { in } \boldsymbol{\Omega}_{\mathrm{s}} \\
\left(\mathbf{a} \cdot\left(\mathbf{e}_{y}\left(\mathbf{U}_{\mathrm{s}}^{1}\right)+\mathbf{e}_{x}\left(\mathbf{U}^{0}\right)\right) \cdot \mathbf{N}=\left(P^{0} \cdot \mathbf{l}+2 M(\omega) \cdot\left(\mathbf{e}_{y}\left(\mathbf{U}_{\mathrm{s}}^{1}\right)+\mathbf{e}_{x}\left(\mathbf{U}^{0}\right)\right)\right) \cdot \mathbf{N}\right. & \text { on } \Gamma \\
\operatorname{grad}_{y}\left(P^{0}\right)+M(\omega) \cdot \mathbf{\Delta}_{y}\left(\mathbf{U}_{\mathrm{f}}^{1}\right)=0 & \text { in } \boldsymbol{\Omega}_{\mathrm{f}} \\
\operatorname{div}_{y}\left(\mathbf{U}_{\mathbf{f}}^{1}\right)+\operatorname{div}_{x}\left(\mathbf{U}^{0}\right)=0 & \text { in } \boldsymbol{\Omega}_{\mathrm{f}} \\
\mathbf{U}_{\mathrm{f}}^{1}=\mathbf{U}_{\mathrm{s}}^{1} & \text { on } \Gamma \\
\operatorname{when}_{x l m}\left(\mathbf{U}^{0}\right)=\left(\delta_{j l} \cdot \delta_{k m}+\delta_{j m} \cdot \delta_{k l}\right) \cdot 1 / 2 &
\end{array}
$$


For the studied geometry, (18) leads, as above, to a linear expression of $U_{\mathrm{s}}^{1}: U_{\mathrm{s} i}^{1}=a_{i} \cdot y_{1}+b_{i}$. On the other hand, the fluid incompressibility implies that:

$$
U_{\mathrm{f} 1}^{1}=a_{1}^{\prime} \cdot y_{1}+b_{1}^{\prime} \quad \text { with } a_{1}^{\prime}=-\operatorname{div}_{x}\left(\mathbf{U}^{0}\right) .
$$

The momentum balance (18.3) gives:

thus:

$$
\mid \begin{aligned}
& d P^{0} / d y_{1}+M(\omega) \cdot d^{2} U_{\mathrm{f} 1}^{1} / d y_{1}^{2}=0 \\
& 2 \cdot M(\omega) \cdot d^{2} U_{\mathrm{f} 2}^{1} / d y_{1}^{2}=0 \\
& 2 \cdot M(\omega) \cdot d^{2} U_{\mathrm{f} 3}^{1} / d y_{1}^{2}=0
\end{aligned}
$$

$$
U_{\mathrm{f} 2}^{1}=a_{2}^{\prime} \cdot y_{1}+b_{2}^{\prime}, \quad U_{\mathrm{f} 3}^{1}=a_{3}^{\prime} \cdot y_{1}+b_{3}^{\prime}, \quad \text { and } \quad P^{0}=P^{0}(x)
$$

The condition of displacement continuity at $y_{1}=0$ together with the periodicity, yield:

$$
b_{i}=b_{i}^{\prime} \quad a_{i}=-a_{i}^{\prime} \cdot n \cdot(1-n)^{-1} .
$$

We still have to express the continuity of the stress at the interface, that is, using the same notations as in Section 3.1.:

$$
\mid \begin{aligned}
& (\lambda+2 \cdot \mu) \cdot a_{1}-\left(P^{0}+2 \cdot M \cdot a_{1}^{\prime}\right)=-\left(2 \cdot(\mu-M) \cdot e_{11}+\lambda \cdot \theta\right) \\
& (\mu-M) \cdot a_{3}^{\prime}=-2 \cdot(\mu-M) e_{12} \\
& (\mu-M) \cdot a_{3}^{\prime}=-2 \cdot(\mu-M) e_{13} .
\end{aligned}
$$

The computation leads to:

$$
\begin{aligned}
a_{1} & =\theta \cdot n \cdot(1-n)^{-1} \\
a_{i} & =-e_{1 i} \cdot 2 \cdot n \cdot(\mu-M) \cdot(n \cdot \mu+(1-n) \cdot M)^{-1} \\
a_{i}^{\prime} & =e_{1 i} \cdot 2 \cdot(1-n)(\mu-M) \cdot(n \cdot \mu+(1-n) \cdot M)^{-1} \quad(i=2,3) \\
P^{0} & =e_{11} \cdot 2 \cdot(\mu-M)+\theta \cdot(\lambda+2 \cdot(n \cdot \mu+(1-n) \cdot M)) \cdot(1-n)^{-1}
\end{aligned}
$$

The vectors $\chi_{j k}^{\prime}$ and the scalars $\tau_{j k}^{\prime}$, are then deduced by indentification, and the value of $C^{\prime * *}$ follows. The matricial form of $C^{\prime * *}$ is more readable:

$$
C^{\prime * *}=\left[\begin{array}{llllll}
C_{11}^{\prime * *} & C_{12}^{\prime * *} & C_{12}^{\prime * *} & & & \\
& C_{22}^{\prime * *} & C_{23}^{\prime * *} & & & \\
& & C_{22}^{\prime * *} & & & \\
& & C_{44}^{\prime * *} & & \\
& & & C_{55}^{\prime * *} & \\
& & & & & C_{55}^{\prime * *}
\end{array}\right] \begin{aligned}
& C_{11}^{\prime * *}=(\lambda+2 \cdot \mu)(1-n)^{-1} \\
& C_{12}^{\prime * *}=(\lambda+n \cdot 2 \cdot \mu)(1-n)^{-1} \\
& C_{23}^{\prime * *}=(\lambda+n \cdot 2(n \cdot \mu+(1-n) \cdot M))(1-n)^{-1} \\
& C_{22}^{\prime * *}=(\lambda+2 \cdot \mu)(1-n)^{-1}+n \cdot 4 \cdot(M-\mu) \\
& C_{44}^{\prime * *}=(1-n) \cdot \mu+n \cdot M \\
& \left.C_{55}^{\prime * *}=2 \cdot \mu \cdot M \cdot(n \cdot \mu+(1-n) \cdot M)\right)^{-1} .
\end{aligned}
$$

\section{CHOICE OF THE BEHAVIOUR FOR A GIVEN PROBLEM}

In the previous sections we pointed out different kinds of behaviour according to the value of $|M(\omega)| /|\mathbf{a}|$ (or $\mu \omega /|\mathbf{a}|$ ) measured by $\varepsilon$. However, for a given material, $L$ depends on the excitation. Thus a given material can present various types of behaviour according to the excitation. Here we propose a criterion for the choice of the suitable behaviour in a problem studied.

In a real case, $M, \mathbf{a}, l$ are known ( $l$ being the dimension of the period or of a representative periodizable volume), but $L$ and consequently $\varepsilon$, remain undetermined at this stage. To impose the value of $\varepsilon$, is not acceptable, because it implicity leads to fix the behaviour of the material arbitrarily. For example when $|M(\omega)| /|\mathbf{a}|=10^{-4}$, the choice $\varepsilon=10^{-2}$ yields to a biphasic behaviour, $\varepsilon=10^{-4}$ yields to an elastic behaviour, and $\varepsilon \ll 10^{-4}$ yields to a viscoelastic behaviour. In order to choose the macroscopic description for a given problem rigorously, we must be in a position to justify the value of $\varepsilon$. Therefore, the selection of the adequate behaviour amounts to a correct estimation of the macroscopic length $L$ associated with the problem. With this aim in view, we make the following reasoning. 
At the first order, the homogenization process, leads to some quantities being constant on the period. For example, $\mathbf{U}_{\mathrm{s}}^{0}$ in all the cases, and $P^{0}$ for the bisphasic medium are only dependent on the variable $x$. Conversely, the results from the homogenization process will be valid only if this condition is effectively satisfied. This means, that if, for example, we focus on the variation of the component $U_{1}^{0}$ of the displacement in the direction $x_{1}$, we must necessarily find:

$$
\left|U_{1}^{0}\left(x_{1}+l\right)-U_{1}^{0}\left(x_{1}\right)\right| /\left|U_{1}^{0}\left(x_{1}\right)\right|=\left|\Delta U_{1}^{0}\right| /\left|U_{1}^{0}\left(x_{1}\right)\right| \leqslant O(\varepsilon)
$$

This result gives a minoration of $\varepsilon$. But, as $\varepsilon$ is the measure of the accuracy of the macroscopic description, the optimum value will be obtained by taking the smallest one allowed, i.e.:

$$
\left|\Delta U_{1}^{0}\right| /\left|U_{1}^{0}\left(x_{1}\right)\right|=O(\varepsilon)
$$

For the sake of simplicity, we put down:

$$
\varepsilon=l / L=\left|\Delta U_{1}^{0}\right| /\left|U_{1}^{0}\left(x_{1}\right)\right|
$$

From this expression, we can now deduce the macroscopic length $L$ associated with a given configuration:

$$
L=l / \varepsilon=l \cdot\left|U_{1}^{0}\left(x_{1}\right)\right| /\left|U_{1}^{0}\left(x_{1}+l\right)-U_{1}^{0}\left(x_{1}\right)\right|=l \cdot\left|U_{1}^{0}\left(x_{1}\right)\right| /\left|\Delta U_{1}^{0}\right| .
$$

Finally, as $l$ is very small with respect to the macroscopic scale:

$$
L=\left|U_{1}^{0}\right| /\left|\left(\partial U_{1}^{0} / \partial x_{1}\right)\right| .
$$

This result leads us to make several remarks:

- The macroscopic length depends on the problem considered. The larger the spatial variations of the macroscopic variables are, the smaller $L$ and the "larger" $\varepsilon$ become. As a consequence, the apparent contrast between the mechanical properties is stronger and the accuracy of the macroscopic description lower.

- On the contrary, the smaller the spatial variations of the macroscopic variables are, the larger $L$ and the smaller $\varepsilon$ become. This corresponds to a smaller contrast of the mechanical properties and a more accurate macroscopic description.

- Let us consider for example an acoustical compression plane wave which propagates in the direction $x_{1}$. The displacement is of the form:

Therefore

$$
U_{1}^{0}=U \cdot \exp (i(\omega \cdot t-k \cdot x))
$$

$$
L=\left|U_{1}^{0}\right| /\left|\partial U_{1}^{0} / \partial x_{1}\right|=|k|^{-1}=2 \cdot \pi / \lambda=\omega / c
$$

where $k, \lambda$, and $c$ are the wave number, the wave length and the celerity of the macroscopic wave, respectively. (We consider here a case where there is no damping.) Consequently, for a harmonic excitation, $\varepsilon=2 \cdot \pi \cdot l / \lambda=l \cdot \omega / c$. Here we again meet the fact that, the nearer the phenomena are close to the diffraction, $(l=O(L))$, the less the homogenization is valid, because of a less clear scale separation $(\varepsilon \approx 1)$.

-For any dynamic excitation, the variations of the response $\mathbf{R}$ in the three directions, lead to a matrix $S_{i j}=R_{i} / R_{i, j}$. The minimum value of $S_{i j}$ (the most unfavorable with regard to the accuracy) gives the macroscopic reference length $L$.

\section{INFLUENCES OF THE VISCOSITY AND OF THE FREQUENCY ON THE DYNAMIC BEHAVIOUR OF A POROUS MEDIUM SATURATED BY A NEWTONIAN FLUID}

With the help of the above determined expression of $\varepsilon$, we can study the evolution of the behaviour due to the variations of the viscosity or the excitation frequency, where all the other characteristics of the medium are kept constant. 


\subsection{Influence of the viscosity}

Let us study how the harmonic behaviour (at a given frequency $\omega_{0}$ ) is modified when the viscosity of the saturating fluid changes. As the frequency of the excitation is supposed to be constant, we can admit, as a first approximation, that the celerity of the wave does not vary too much with the fluid viscosity, and the value of the small parameter $\varepsilon$ can be reasonably considered as a constant $\varepsilon_{0}=l \cdot \omega_{0} / c$ independent of the viscosity. Thus we see that for viscosity values close to $\mu_{0}=|\mathfrak{a}| / \omega, \mu_{1}=\varepsilon \cdot \mu_{0}$, or $\mu_{2}=\varepsilon^{2} \cdot \mu_{0}$, the behaviour will be respectively viscoelastic, elastic or biphasic. The behaviour is thus clearly defined in three viscosity ranges. But we remark that if $\varepsilon$ is very small, the values $\mu_{0}, \mu_{1}, \mu_{2}$, are very different. So the question of the selection of the appropriate behavior for intermediate viscosities still remains. (For example if $\varepsilon=10^{-6}$ and $\mu \omega /|\mathbf{a}|=10^{-3}$ must we select the elastic or the viscoelastic behaviour?) We show below that in fact, there is a continuous evolution from the biphasic to the elastic and then to the viscoelastic model.

5.1.1 Continuous passage from the biphasic to the elastic behaviour. We suppose here that the saturating fluid has a viscosity $\mu=m \cdot \mu_{2}$ so that for $m$ equal to 1 the macroscopic behaviour is biphasic. We then prove that when $m$ increases from 1 to $\varepsilon^{-1}$, the behaviour changes progressively from biphasic to elastic.

Assuming $m$ close to 1 , the results of Section 2.3.1 can be applied, and we obtain macroscopic behaviour where the Darcy law is written in the following form:

$$
\begin{aligned}
\left\langle\mathbf{U}_{\mathrm{f}}\right\rangle-n \cdot \mathbf{U}_{\mathrm{s}} & =\left(\mathbf{K}\left(\mu, \omega_{0}\right) / i \mu \omega_{0}\right) \cdot\left(\operatorname{grad}(P)+\rho_{f} \cdot \omega^{2} \mathbf{U}_{\mathrm{s}}\right) \\
& =\left(\mathbf{K}\left(\mu, \omega_{0}\right) / i \omega_{0} m \cdot \mu_{2}\right) \cdot\left(\operatorname{grad}(P)+\rho_{f} \cdot \omega^{2} \mathbf{U}_{\mathrm{s}}\right) .
\end{aligned}
$$

As we increase the viscosity, i.e. $m$, the weight of the inertial effects at the microscopic level, becomes more and more weak. And when $m$ tends to $\varepsilon^{-1}$, i.e. $\mu \approx \mu_{1}$, we have $\mathbf{K}\left(\mu, \omega_{0}\right) \cdots \mathbf{k}$, with $\mathbf{k}=O\left(l^{2}\right)$ and consequently:

$$
\mathbf{K}\left(\mu, \omega_{0}\right) / \omega_{0} m \cdot \mu_{2} \cdots \varepsilon^{-1} \cdot \mathbf{k} / \omega_{0} \cdot \mu_{0}=O(\varepsilon) .
$$

The magnitude of the relative motion between the fluid and the solid is of the order $\varepsilon$ and therefore they are negligible at the first order at the macroscopic level. Then the medium becomes monophasic, and the compatibility equation based on the volume balance degenerates into:

$$
0=-\boldsymbol{\alpha} \cdot \mathbf{e}\left(\mathbf{U}_{\mathrm{s}}\right)+\beta \cdot P .
$$

Therefore, when $m$ tends to $\varepsilon^{-1}$ the macroscopic description becomes:

$$
\begin{aligned}
& \langle\boldsymbol{\sigma}\rangle=\mathbf{C} \cdot \mathbf{e}\left(\mathbf{U}_{\mathrm{s}}\right)+\alpha \cdot P \\
& \operatorname{div}\langle\boldsymbol{\sigma}\rangle=-(1-n) \cdot \rho_{\mathrm{s}} \cdot \omega^{2} \mathbf{U}_{\mathrm{s}}-n \cdot \rho_{f} \cdot \omega^{2}\left\langle\mathbf{U}_{\mathrm{f}}\right\rangle \\
& \left\langle\mathbf{U}_{\mathrm{f}}\right\rangle-n \cdot \mathbf{U}_{\mathrm{s}}=0 \\
& 0=-\boldsymbol{\alpha} \cdot \mathbf{e}\left(\mathbf{U}_{\mathrm{s}}\right)+\beta \cdot P .
\end{aligned}
$$

This behaviour is the monophasic elastic one introduced in Section 2.3.2.

In conclusion when the viscosity increases, the relative fluid-solid displacement, restricted by the viscosity, becomes weaker, until it is of the same order as the intrinsic errors due to the homogenization: It must be considered as null. The biphasic behaviour degenerates progressively to an elastic behaviour.

5.1.2 Continuous passage from the viscoelastic to the elastic behaviour. We now assume that the saturating fluid has a viscosity $\mu=m \cdot \mu_{0}$ so that for $m$ equal to 1 the macroscopic behaviour is viscoelastic. We then show that when $m$ decreases from 1 to $\varepsilon$, the behaviour changes progressively from viscoelastic to elastic.

Assuming $m$ close to 1 , let us come back to Section 2.3.3. The second problem is now 
modified to:

$$
\begin{array}{ll}
\mathbf{U}_{\mathrm{s}}^{1}(x, y), \mathbf{U}_{\mathrm{f}}^{1}(x, y) \quad \text { and } P^{0}(x, y) & \Omega \text {-periodic in } y . \\
\operatorname{div}_{y}\left(\mathbf{a} \cdot\left(\mathbf{e}_{y}\left(\mathbf{U}_{\mathrm{s}}^{1}\right)+\mathbf{e}_{x}\left(\mathbf{U}^{0}\right)\right)\right)=0 & \text { in } \Omega_{\mathrm{s}} \\
\left(\mathbf{a} \cdot\left(\mathbf{e}_{y}\left(\mathbf{U}_{\mathrm{s}}^{1}\right)+\mathbf{e}_{x}\left(\mathbf{U}^{0}\right)\right)-P^{0} \cdot l\right) \cdot \mathbf{N}=2 i \omega m \mu_{0} \cdot\left(e_{y}\left(\mathbf{U}_{\mathrm{f}}^{1}\right)+e_{x}\left(\mathbf{U}^{0}\right)\right) \cdot \mathbf{N} & \text { on } \Gamma \\
\operatorname{grad}_{y}\left(P^{0}\right)=-2 i \omega m \mu_{0} \cdot \Delta_{y}\left(\mathbf{U}_{\mathrm{f}}^{1}\right) & \text { in } \Omega_{\mathrm{f}} \\
\operatorname{div}_{y}\left(\mathbf{U}_{\mathrm{f}}^{1}\right)+\operatorname{div}_{x}\left(\mathbf{U}^{0}\right)=\mathbf{0} & \text { in } \Omega_{\mathrm{f}} \\
\mathbf{U}_{\mathbf{f}}^{1}=\mathbf{U}_{\mathrm{s}}^{1} & \text { on } \Gamma
\end{array}
$$

from which the solution is:

$$
\mathbf{U}_{\mathrm{s}}^{1}=\mathbf{u}^{1}(x)+\chi_{j k}\left(y, m \mu_{0} \omega_{0}\right) e_{x j k}\left(\mathbf{U}^{0}\right) \quad P^{0}=\tau_{j k}\left(y, m \mu_{0} \omega_{0}\right) \cdot e_{x j k}\left(\mathbf{U}^{0}\right) .
$$

If we decrease the viscosity, i.e. if $m$ tends to $\varepsilon$, the right hand members of (19) become negligible, and we get:

$$
\mathbf{U}_{\mathrm{s}}^{1}=\mathbf{u}^{1}(x)+\chi_{j k}(y, 0) e_{x j k}\left(\mathbf{U}^{0}\right) \quad P^{0}=\tau_{j k}(y, 0) \cdot e_{x j k}\left(\mathbf{U}^{0}\right)=\tau_{j k}^{0} \cdot e_{x j k}\left(\mathbf{U}^{0}\right) .
$$

Moreover, equations (19b) and (19c) which lead to the determination of $\mathbf{U}_{\mathrm{s}}^{1}$, become identical to those allowing the determination of $\mathbf{U}_{\mathrm{s}}^{1}$ in the case of an elastic macroscopic behaviour [set (9)]. Therefore we can write:

$$
\mathbf{U}_{\mathrm{s}}^{1}=\mathbf{u}^{1}(x)+\chi_{j k}(y, 0) e_{x j k}\left(\mathbf{U}^{0}\right)=\mathbf{u}^{1}(x)+\xi_{j k}(y) \cdot e_{x j k}\left(\mathbf{U}^{0}\right)+\eta(y) \cdot P^{0}
$$

which leads to the following relation between the "viscoelastic" and the "elastic" fields:

$$
\boldsymbol{x}_{j k}(y, 0)=\xi_{j k}(y)+\eta(y) \cdot \tau_{j k}^{0} \text {. }
$$

The expression of $\mathbf{U}_{\mathrm{s}}^{1}$ being identical to that obtained in the elastic case, it remains to be proved that the pressure is also unchanged. To this end, let us integrate equation (19d) over $\Omega_{\mathrm{s}}$, and use (19e):

$$
\int_{\Omega_{\mathrm{f}}} \operatorname{div}_{x}\left(\mathbf{U}^{0}\right) \mathrm{d} \Omega=-\int_{\mathbf{\Omega}_{\mathrm{f}}} \operatorname{div}_{y}\left(\mathbf{U}_{\mathrm{f}}^{1}\right) \mathrm{d} \Omega=-\int_{\Gamma} \mathbf{U}_{\mathrm{f}}^{1} \cdot \mathbf{N}_{\mathrm{f}} d \mathrm{~s}=\int_{\Gamma} \mathbf{U}_{\mathrm{s}}^{1} \cdot \mathbf{N}_{\mathrm{s}} d \mathrm{~s}=\int_{\Omega_{\mathrm{s}}} \operatorname{div}_{y}\left(\mathbf{U}_{\mathrm{s}}^{1}\right) \mathrm{d} \Omega
$$

and, according to (20):

$$
\int_{\Omega_{\mathrm{f}}} \operatorname{div}_{x}\left(\mathbf{U}^{0}\right) \mathrm{d} \Omega=\int_{\Omega_{\mathrm{s}}} \operatorname{div}_{y}\left(\xi_{j k}(y) \cdot e_{x j k}\left(\mathbf{U}^{0}\right)+\boldsymbol{\eta}(y) \cdot P^{0}\right) \mathrm{d} \Omega .
$$

Regrouping the different terms, we obtain:

$$
\boldsymbol{\alpha} \cdot \mathbf{e}_{x}\left(\mathbf{U}^{0}\right)=\beta \cdot P
$$

which effectively relates the pressure to the deformations in the elastic case, and gives the relation:

$$
\tau_{i j}^{0}=\alpha_{i j} \cdot \beta^{-1} \text {. }
$$

Thus we observe that the microscopic field in the viscoelastic case, tends to the elastic case one, when the viscosity decreases. This comes from the fact that the fluid shear stresses in the volume $\Omega_{\mathrm{f}}$ and at the interface $\Gamma$ become of the same order as the one due to the intrinsic error of the method and must be neglected. When the viscosity decreases, the viscoelastic macroscopic description changes progressively into an elastic description.

5.1.3 Conclusion. The macroscopic dynamic behaviour of a porous saturated medium at a constant frequency $\omega_{0}$ changes continuously from biphasic to elastic, and to viscoelastic, when the viscosity of the saturating fluid increases. The elastic state can be interpreted as a limit case either of the biphasic state, either of the viscoelastic state. Consequently, for intermediate viscosities it is more judicious to use the more informative description (i.e. biphasic or viscoelastic), while keeping in mind the accuracy of the homogenized model.

This reasoning, which is developed for the case of a Newtonian fluid is also valid for a viscoelastic fluid (when the dispersive effects evoked in Section 2.4.1 stay weak). 


\subsection{Influence of the frequency}

Let us now study how the behaviour of a porous medium saturated by a same Newtonian fluid, changes with the frequency. On the contrary to the previous case, $\varepsilon=2 \cdot \pi \cdot l / \lambda$ is no more constant, and the behaviour is characterized by the value of the exponent $x$ defined by:

$$
\begin{array}{ll}
\mu \omega /|\mathbf{a}|=\varepsilon^{x} & \begin{array}{l}
0<x<1: \text { viscoelastic behaviour } \\
x=1: \text { elastic behaviour } \\
1<x<2: \text { biphasic behaviour. }
\end{array}
\end{array}
$$

If we focus on the weakly dispersive waves, such as the first compressional and the shear waves (the second compressional wave in the biphasic case is excepted), we can reasonably consider the wave celerities as constants all over the concerned frequency range. Therefore we have: $\lambda=c / f$ that is also: $\omega=2 \cdot \pi \cdot c / \lambda=c / L=\varepsilon \cdot c / l$.

Introducing this expression of $\omega$ in (21), $x$ is the solution of the following equation:

$$
\varepsilon \cdot \mu c / l \cdot|\mathbf{a}|=\varepsilon^{x}, \quad \text { or: } \quad(x-1) \cdot \log (\varepsilon)=\log (\mu c / l \cdot|\mathbf{a}|) .
$$

Since $\varepsilon<1$ (if not, homogenization is not valid), $(x-1)$ and $\log (\mu c / l \cdot|\mathbf{a}|)$ are of opposite sign, two cases appear:

1 Material such as $\mu c / l \cdot|\mathbf{a}|>1$. Whatever the frequency is (the domain of the diffraction again is excepted), we have $x-1<0$. More precisely we get:

$$
x=1+\log (\mu c / l \cdot|\mathbf{a}|) / \log (\omega \cdot l / c) \text {. }
$$

Examining this expression of $x$, we first observe that when decreasing the frequency, the viscoelastic behaviour $(x<1)$ turns into elastic behaviour which is reached when the frequency vanishes $(w \ldots 0 ; x \rightarrow 1)$. And secondly we see that biphasic behaviour is not possible for such medium.

2 Material such as $\mu c / l \cdot|\mathbf{a}|<1$. In this case, for the range of frequency where the homogeneization is valid, we have $x-1>0$. Expression (22) shows that when decreasing the frequency, the biphasic behaviour $(x=2)$ is progressively changed into elastic behaviour which is reached when the frequency tends to zero. This effectively corresponds to the features observed concerning the $P_{1}$ or $S$ waves: in the low frequency range the relative motion between fluid and solid is not stimulated.

If we focus now on the second compressional wave $P_{2}$, which exists only in the biphasic material, the above results stay valid in the high frequency range, but must be modified for the low frequencies because of the strong dispersivity of this wave.

At the low frequency we have [12]:

$$
\lambda_{P_{2}}=2 \pi \cdot \sqrt{2|\mathbf{k}| \cdot|\mathbf{a}| / \omega \mu}, \quad \text { thus: } \quad \varepsilon=2 \cdot \pi \cdot l / \lambda=\sqrt{\mu \omega /|\mathbf{a}|} \cdot \sqrt{1 / 2|\mathbf{k}|} .
$$

Since $|\mathbf{k}|=O\left(l^{2}\right)$, we always obtain $\mu \omega /|\mathbf{a}|=O\left(\varepsilon^{2}\right)$ for the $P_{2}$ wave and the behaviour is biphasic This result is in agreement with a main feature of this wave: the relative motion between the two phases, is always stimulated whatever the frequency is.

Thus for this kind of medium, according to the macroscopic excitation, the behaviour can be, either biphasic all over the frequency range $\left(P_{2}\right.$ wave), or biphasic (at high frequency) and then monophasic elastic (at low frequency) (for the $P_{1}$ and $S$ waves). In all cases the viscoelastic behaviour is not reached.

\section{STUDY OF THE BITUMINOUS CONCRETE}

For the investigation of the behaviour of a porous medium saturated by a viscoelastic fluid at a given frequency $f$, we only need the value of $M(\omega)$ (cf. 1.4.). Now if we want to follow the change of this behaviour according to the frequency, we have to make the evolution of $M$ with respect to the frequency precise, i.e. introduce the rheology of the viscoelastic fluid. For example the variations of $M(\omega)$ for a Maxwell fluid are not the same as the bitumen one, so the changes of the macroscopic model in these both cases are different. 
In this section, we propose to investigate the bituminous concrete as a mixture of solid grains and bitumen. The study of this material is interesting for two reasons:

-First, the bituminous concrete is a materal commonly used in the road construction, and has been extensively studied in both experimental and theoretical ways.

-Secondly, the variability over several decades, of the mechanical properties of the bitumen, in the usual temperature range, leads us to forecast significant variations of the mixture behaviour at the macroscopic scale.

After a recall of the main mechanical features concerning the bitumen and bituminous concrete, we study the evolution of the macroscopic behaviour according to the temperature and the frequency. Then we make the range of validity of the time-temperature equivalency principle when applied to the mixture precise. Finally a qualitative comparison, between experimental results and the undirectional model studied above, is presented.

\subsection{Experimental behaviour of the bitumen and bituminous concrete}

Let us recall the properties of the bitumen and bituminous concrete, obtained by vibratory experiments [2], [15], [16], [20], [21].

6.1.1 Behaviour of the bitumen. Testing the bitumen with small harmonic strains, one observes a viscoelastic behaviour. Plotted in the Cole and Cole plan $\left(M_{r}, M_{i}\right)$, the experimental values of the complex modulus, at a given temperature $T$ and for various frequencies, describe a curve parametrized by the frequency (see Fig. 3). Moreover, by testing the bitumen, at a given frequency $f$ for various temperatures, ones notes that the complex modulus describes exactly the same curve (Fig. 3 now parametrized by the temperature). This fact shows that the bitumen satisfies a time-temperature equivalency principle in the range investigated by the experiments $\left(0<f<1 \mathrm{kHz} ; 0<T<80^{\circ} \mathrm{C}\right)$. Thereby, the viscoelastic modulus only depends on a reduced variable $\omega \cdot \tau$ where $\omega=2 \pi \cdot f$ is the pulsation of the excitation, and $\tau$ a characteristic time. One shows that $\tau$ only depends on the temperature by the William-Landel and Ferry law: $\tau_{T}=\tau_{0} \cdot a_{0}(T)$ where the translation factor $a_{0}(T)$ is given by:

$$
\log \left(a_{0}(T)\right)=C_{1}\left(T-T_{0}\right) /\left(C_{2}+T-T_{0}\right)
$$

and $T_{0}$ is a temperature of reference.

For numerous hydrocarbonate materials, the constants $C_{1}$ and $C_{2}$, are given with a good accuracy, by: $C_{1}=-8.86, C_{2}=101.6$ when the reference temperature $T_{0}$ is equal to: $T_{0}=T_{\mathrm{g}}+50^{\circ} \mathrm{C} ; T_{\mathrm{g}}$ corresponding to the temperature on the vitrification point $\left(T_{\mathrm{g}} \approx-15^{\circ} \mathrm{C}\right.$ for the bitumens).

In other respects, the asymmetrical curve plotted in Fig. 3 can be approximated with a very good agreement, by an expression of the complex modulus of the following form [15]:

$$
M(\omega \tau)=M^{\infty} \cdot\left(1+\delta \cdot(i \omega \tau)^{-k}+(i \omega \tau)^{-h}\right)^{-1}
$$

where $M^{\infty}, \delta, k, h$, depend on the investigated bitumen.

Remarks

When $\omega \tau$ tends to infinity (i.e. at low temperature or high frequency), the bitumen behaves as an elastic solid having a rigidity $M^{\infty}$. At the opposite, when $\omega \tau$ is small, (i.e. at high

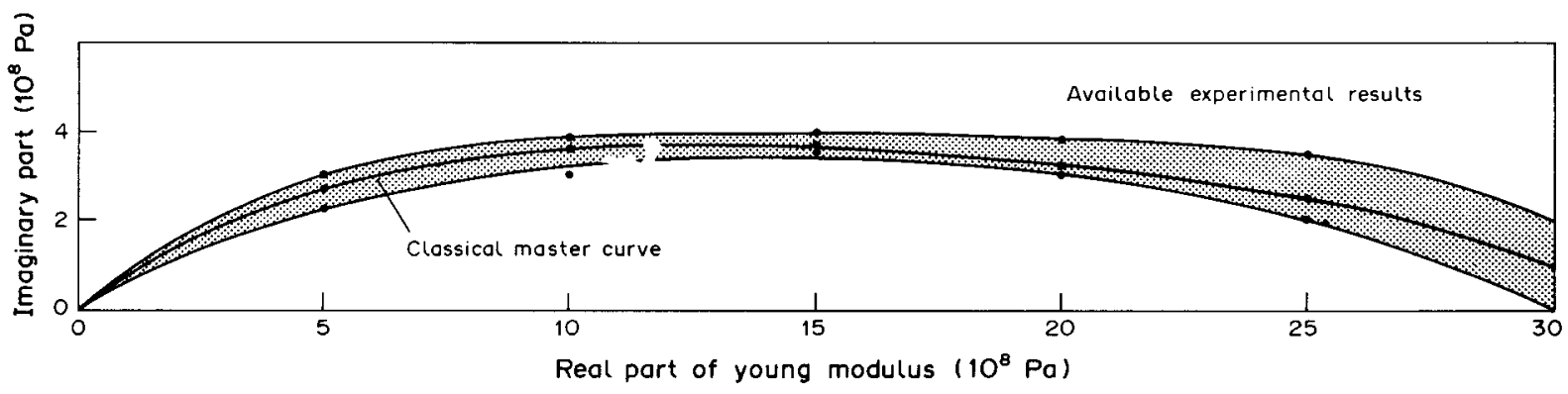

Fig. 3. Complex modulus of bitumen (from Sayegh [20]). Horizontal axis, Real part of Young modulus $\left(10^{8} \mathrm{~Pa}\right)$. Vertical axis, Imaginary part $\left(10^{8} \mathrm{~Pa}\right)$. Main field of available experimental results and classical master curve. 
temperature or low frequency), the behaviour remains that of a viscoelastic fluid $(M \approx$ $\left.M^{\infty}(i \omega \tau)^{h}\right)$. The range of the very low values of $\omega \tau$ is not reached by the vibratory experiments published in the literature, but different experimental tests prove that for temperature close to $150^{\circ} \mathrm{C}$, the bitumen becomes a Newtonian fluid, $(\mu \approx 0.1 \mathrm{~Pa} \mathrm{~s})$. Then expression (23) is no longer valid.

6.1.2 Experimental behaviour of the bituminous concrete. The bituminous concrete used in civil engineering, is a mixture of solid grains, bitumen and air. As the degree of saturation in bitumen is very high $(95 \%)$, this gaseous phasis is neglected afterwards. We here relate some experimental results reported in [15], [20].

This material, undergoing small vibratory strains, behaves mainly as a viscoelastic solid, and it seems that a time-temperature equivalency principle might be respected. As a general rule, in the Cole and Cole plan, the modulus follows a master curve similar to the one presented in Fig. 4. Roughly, we can separate three kinds of behaviour:

-At low temperature and high frequency, the behaviour is elastic with a modulus $E^{\infty}$.

-At high temperature and low frequency, generally speaking, the material tends to another elastic state with a weak static rigidity $E^{0}$. (In some cases, particularly when the dimensions of the grains and their concentration is weak, this static modulus is null and the behaviour remains viscoelastic [21]).

-For the intermediate values of temperature and frequency, the behaviour is viscoelastic. In the literature, there is no mention of biphasic behaviour.

We compare in the next section these experimental facts to the theoretical approach derived from the homogenization.

\subsection{Homogenized behaviour of the bituminous concrete: influences of the frequency and the temperature}

Let us apply the results of the homogenization in order to specify the behaviour according to the frequency and the temperature. Following the same method as the one used for a Newtonian saturating fluid, the kind of behaviour is fixed by the value of the exponent $x$ defined by:

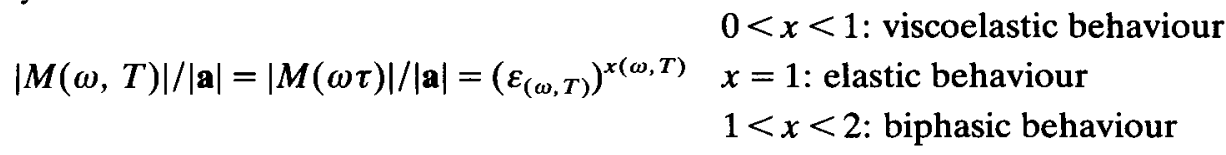

The determination of $x(\omega, T)$ for any couple $\omega, T$, is difficult for two reasons:

-On the one hand, the expression of $M(\omega, \tau)$ is intricated.

-On the other hand, the dispersive effects can no longer be neglected, since the experiments point out variations of modulus from $5 \cdot 10^{7}$ to $2 \cdot 10^{10} \mathrm{~Pa}$, which leads to wave's celerities from 200 to $2500 \mathrm{~m} / \mathrm{s}$.

Nevertheless, if we only want to determine the ranges of viscoelastic or biphasic behaviour, it is sufficient to draw the boundary curve in the plan $(\omega, T)$ delimiting the elastic state $(x(\omega, T)=1)$. In this aim, we have to find the couples of values $(\omega, T)$ such as:

$$
|M(\omega, T)| /|\mathbf{a}|=|M(\omega \tau)| /|\mathbf{a}|=\varepsilon \quad \text { with } \varepsilon=\omega \cdot l / c^{0},
$$

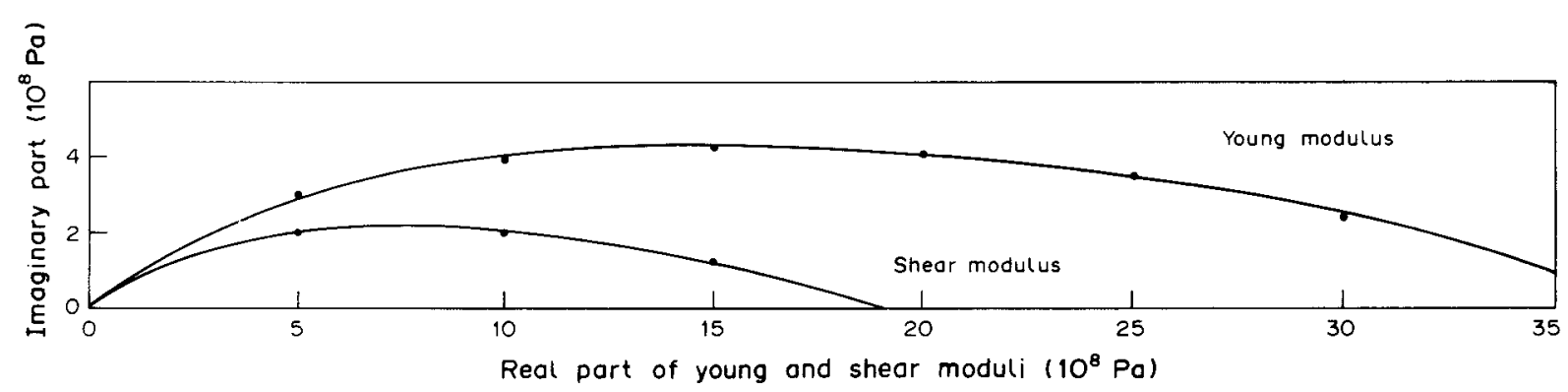

Fig. 4. Complex coefficient of bituminous concretes. Horizontal axis, Real part of Young and shear moduli $\left(10^{8} \mathrm{~Pa}\right)$. Vertical axis, Imaginary part $\left(10^{8} \mathrm{~Pa}\right)$. 
where $c^{0}$ is the wave's celerity of the macroscopic elastic equivalent medium (with $C^{*}$ as elastic tensor).

The expression of $M(\omega \tau)$ does not allow us to solve equation (24) analytically. Consequently we compute the solution numerically for a particular case. The properties of the materials introduced in the calculations are given in the table below, and correspond to very common features.

Bitumen

\section{Solid grains}

$$
\begin{gathered}
M(\omega \tau)=10^{9} \cdot\left(1+2(i \omega \tau)^{-0.2}+(i \omega \tau)^{-0.6}\right)^{-1}\left(\mathrm{~N} / \mathrm{m}^{2}\right) \\
\rho_{\mathrm{f}}=1007\left(\mathrm{~kg} / \mathrm{m}^{3}\right) \\
2 \pi \cdot \tau_{15^{\circ} \mathrm{C}}=10^{-2}(\mathrm{~s})
\end{gathered}
$$

\section{Bituminous concrete}

$$
\mu_{s}=10^{10}\left(\mathrm{~N} / \mathrm{m}^{2}\right) \text { (shear rigidity) } \quad \rho_{s}=2600\left(\mathrm{~kg} / \mathrm{m}^{3}\right)
$$

$$
\begin{array}{ll}
n=0.25 & l=10^{-2}(\mathrm{~m}) \\
G^{\infty}=10^{10}\left(\mathrm{~N} / \mathrm{m}^{2}\right) & c^{\infty}=2100(\mathrm{~m} / \mathrm{s}) \\
G^{0}=9 \cdot 10^{7}\left(\mathrm{~N} / \mathrm{m}^{2}\right) & c^{0}=200(\mathrm{~m} / \mathrm{s})
\end{array}
$$

The solving method is simple: for a given value $z$ of $\omega \tau$, the ratio $|M(z)| / \mu_{\mathrm{s}}$ gives $\varepsilon$, from which we deduce:

$$
\omega=\varepsilon \cdot\left(c^{0} / l\right)=\left(|M(z)| / \mu_{\mathrm{s}}\right) \cdot c^{0} / l,
$$

then $\tau=z / \omega$ and endly, $T$ by inversing the William, Landel and Ferry law. The results are presented on the plan $(\log (\tau), \log (\omega))$ or $(T, \log (\omega))$ (see Fig. 5).

\section{Discussion}

As opposed to the case of a Newtonian saturating fluid, we remark that, if we carry out tests at high temperature ( $\tau$ constant, small) for various frequencies, the material passes through the

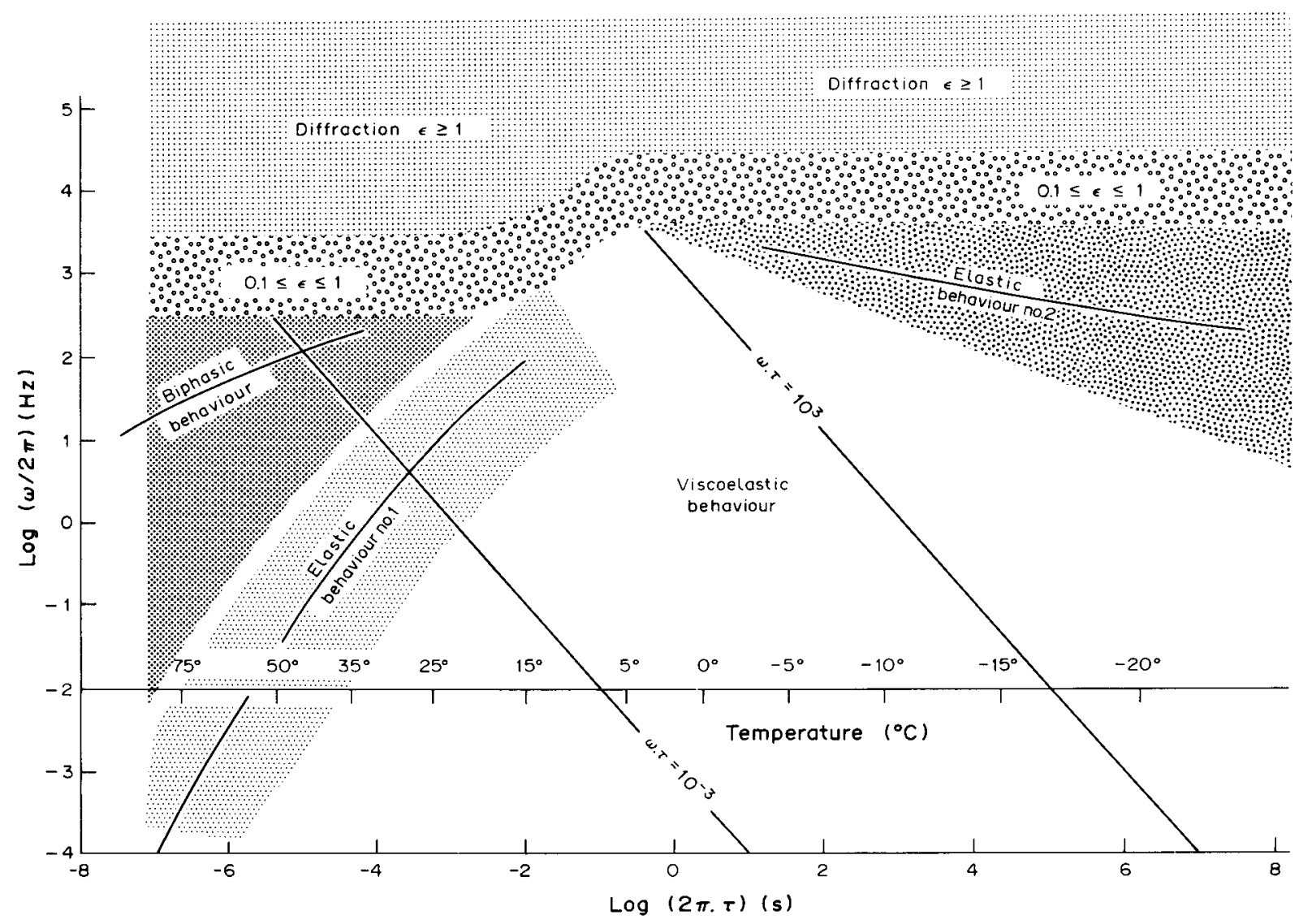

Fig. 5. Different types of behaviour of the bituminous concrete according to the temperature and the frequency. Horizontal axis, $\log$ of characteristic time: $\log (2 \pi \tau)(\mathrm{s})$. Vertical axis, $\log$ of the frequency: $\log (\omega / 2 \pi)(\mathrm{Hz})$. 
three kinds of behaviour: as a matter of fact, for small values of $\omega \tau,|M| \approx M^{\infty} \cdot(\omega \tau)^{0.6}$, so $|M| /|\mathbf{a}|$ changes more slowly than $\varepsilon$. Thus, decreasing the frequency from a biphasic state, $x$ decreases until it passes beyond the value of 1 (elastic state) and reaches the viscoelastic behaviour.

In the same way, testing the material at a given frequency, for various temperatures, one would observe an evolution from the biphasic behaviour (high temperatures) to the viscoelastic behaviour (low temperatures).

Moreover, concerning the large values of $\omega \tau$ (high frequencies, low temperatures), the bituminous concrete behaves as an elastic medium, since the imaginary part of the complex modulus becomes negligible (say $O(\varepsilon)$ ) in comparison with the real part. Then, we are in accordance with the remark in Section 2.4.3: The bituminous concrete presents a second elastic behaviour (noted $\mathrm{n}^{\circ} 2$ as opposed to $\mathrm{n}^{\circ} 1$ seen above). In Fig. 5, we plot the curve bounding this second elastic range, which has been obtained by solving the following equation:

$$
M_{i}(\omega \tau)=M_{r}(\omega \tau) \cdot \varepsilon_{(\omega, \tau)} \text { with } \varepsilon=\omega \cdot l / c^{\infty},
$$

$c^{\infty}$ being the velocity obtained from the characteristics at high frequency (large $\omega \tau$ ) of the macroscopic continuum $\left(\mathbf{C}^{\prime * *}\right)$.

Finally, we indicate on Fig. 5 the limit frequencies corresponding to the diffraction $(\varepsilon=1)$ and the limit of validity of the homogenization $(\varepsilon=0.1)$.

The qualitative description of the behaviour evolution from elasticity to viscoelasticy and once again to an other elasticity, is in agreement with the experimental results described above. The biphasic effects happen to be outside of the experimental range investigated up to now. But the sweating of the bituminous concrete, which is commonly observed in situ during hot weather, effectively seem to match these phenomena.

\subsection{Time-temperature equivalency principle for the bituminous concrete}

Can we extend the time-temperature equivalency principle to the bituminous concrete? As the viscoelastic, elastic and biphasic models can never be equivalent, it is obvious that if a time-temperature equivalency principle subsists at the macroscopic scale, its application will necessarily be restricted to each domain of behaviour.

6.3.1. Viscoelastic behaviour (inclusing the second kind of elasticity). Coming back to the homogenization presented in Section 2.4.3, it appears that, since $M$ only depends on $\omega \tau$, the viscoelastic tensor $\mathbf{C}^{\prime * *}$ also depends only on this reduced frequency. A priori, this remark leads us to conclude that the time-temperature equivalency principle still holds at the macroscopic level. Moreover the translation factor $a_{0}(T)$ is the same for the bituminous concrete as for the bitumen.

Nevertheless, as $\mathbf{C}^{\prime * *}$ is constant for a given value of $\omega \tau$ (and consequently the velocity of the different waves), the magnitude of $\varepsilon$ which measures the accuracy of the macroscopic description changes with the frequency of the excitation. So the above result must be applied cautiously. For example if the coefficients of the homogenized behaviour are obtained with an accuracy of $10^{-3}$ at $1 \mathrm{~Hz}$ (for a temperature $T_{1}$ ), then the same coefficients could only be measured with an accuracy of $10^{-1}$ at $100 \mathrm{~Hz}$ (for a temperature $T_{2}$ such that the time-temperature equivalency principle is satisfied: $\tau_{T_{2}}=10^{-2} \cdot \tau_{T_{1}}$ ).

As a conclusion, the time-temperature equivalency principle remains valid in the viscoelastic range, but only in a limited interval of frequency, the factor of translation for the bituminous concrete being the same as the one of the bitumen.

6.3.2 Elastic behaviour. We saw in Section 2.4.2 that the elastic behaviour is unchanged whatever the rheology of the fluid. That means that $\mathbf{C}^{*}$ does not depend on $\omega \tau$. The elasticity of the skeleton masks the viscoelastic properties of the fluid, and in this case the time-temperature equivalency principle looses its sense at the macroscopic level.

6.3.3 Biphasic behaviour. As previously, for a biphasic medium, $\mathbf{C}$ does not depend on $\omega \tau$. Inversely, the generalized Darcy tensor $\mathbf{K}^{\prime}$ depends both on $M(\omega \tau)$ and $\omega$. So the filtration law remains unchanged on the only condition that $M$ and $\omega$ remain constant. This necessarily implies to fix the temperature and the frequency: generally speaking, the time-temperature equivalency principle is no longer valid in the biphasic state. 


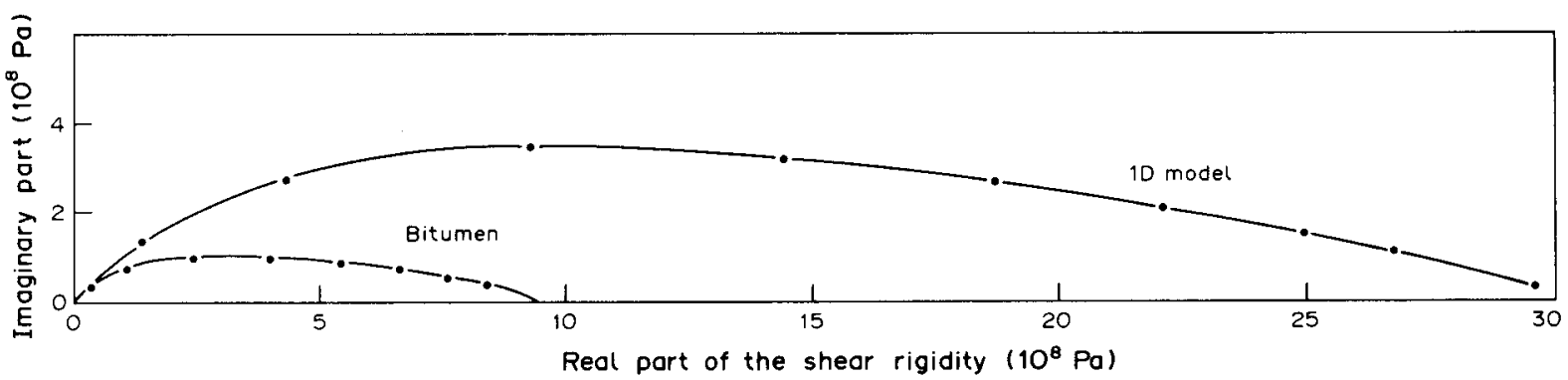

Fig. 6. Complex rigidity coefficient $C_{55}^{\prime * *}$ of the $1 \mathrm{D}$ model. Horizontal axis, Real part of the shear rigidity $\left(10^{8} \mathrm{~Pa}\right)$. Vertical axis, Imaginary part $\left(10^{8} \mathrm{~Pa}\right)$.

Nevertheless, if we study frequencies low enough to neglect the inertial effects at the microscopic scale, $\mathbf{K}^{\prime}$ tends to the intrinsic permeability, which is independent of the rheology of the fluid. So the Darcy law becomes:

$$
\left\langle\mathbf{U}_{\mathbf{f}}\right\rangle-n \cdot \mathbf{U}_{\mathrm{s}}=(\mathbf{k} / M) \cdot \operatorname{grad}(P)
$$

Under these conditions, keeping in mind the restrictions related to the accuracy, the time-temperature equivalency principle still holds for the filtration law, with the same factor of translation that the one of the bitumen.

\subsection{Qualitative comparison with the unidirectional model}

In paragraph 6.2 , we have shown that the homogenization leads to results which are in agreement with the experiments, at least inside the explored range of excitations. An accurate quantitative approach needs the definition of a representative cell of the material, and then the calculation of the macroscopic coefficients associated with this period.

However, in a first step, we can try to simulate the modulus of the bituminous concrete under shear strains, by using the 1D model presented in Section 3. Therefore, we draw the variation of the coefficient $\mathrm{C}_{55}^{\prime * *}$ according to $\omega \tau$ (see Fig. 6), in the Cole and Cole plan. This coefficient corresponds to the shear rigidity in the plans of the layers, and can be representative of the effects due to the joints of bitumen between the solid grains. The microscopic parameters introduced in this investigation, are those used in Section 6.2.

We observe that the coefficients calculated are not directly transposable to a common bituminous concrete. On the one hand, there is no static elasticity. On the other hand, the shear modulus at high frequencies-low temperatures, is weaker as usual. These misfeats, essentially come from the unidirectional structure of the cell.

But, the general aspect of the curve is consistent with the one observed on the real materials. This results allows us to expect good simulations from a more realistic microscopic geometry. (Particularly, with a connected skeleton and pores in the three directions of the space.)

\section{CONCLUSION}

In this study based on the homogenization technique, we pointed out that the porous media saturated either by a Newtonian fluid or by a viscoelastic fluid behaves as a biphasic, elastic or viscoelastic material depending of the excitation. The kind of behaviour is determined by the amplitude of the contrast between the mechanical properties of the both constituants, the measure of the contrast being made using the powers of $\varepsilon$.

In the acoustic field, $\varepsilon$ is directly related to the wave length of the macroscopic phenomena studied. Consequently, the description of the medium changes with the frequency.

These results applied to the bituminous concrete, are in agreement with the experiments. Moreover, the homogenization procedure allows us to give the accuracy of the equivalent continuum medium, and fix the limits of the validity of the time-temperature equivalency principle at macroscopic level. 


\section{REFERENCES}

[1] R. J. ATKIN and R. E. CRAINE, Continuum theories of mixtures: applications. J. Inst. Math. Appl. 17, 153-207 (1976).

[2] P. ATTANE, A. SOUCEMARIANADAN, G. TURREL and J. B. PRUD'HOMME, Non linear behaviour of asphalts in steady and transient shear flow. Rheological Acta 23, 297-310.

[3] D. AUBRY, E. BARD and A. MODARESSI, Mixture approach to the mechanical behaviour of the bituminous concrete. Constitutive laws for Engineering Materials (Edited by D. DESAI et al.). Elsevier, Tucson (1987).

[4] J. L. AURIAULT, Dynamic behaviour of a porous medium saturated by a newtonian fluid Int. J. Engng Sci. 18, $775-785$ (1980).

[5] J. L. AURIAULT, Homogenization-application to porous saturated media In Two phase Medium Mechanics, Summer School Gdansk, 1983. Publication de l'Institut Polytechnique de Gdansk.

[6] J. L. AURIAULT and G. BONNET, Dynamiques des composites élastiques périodiques. Arch. Mech. 37, (4-5) 269-284, Warszawa (1985).

[7] J. L. AURIAULT and D. CAILLERIE, Quelques remarques sur les methodes d'homogénéisation. Revue Française de géotechnique (1988).

[8] J. L. AURIAULT, Dynamic behaviour of porous media. N.AT.O.A.S.I.: Transport Process in Porous Media, Washington State University, U.S.A., July 1989. (To be published) Kluwer Academic Press.

[9] J. BEAR, Dynamics of Fluids in Porous Media. American Elsevier, New York (1972).

[10] A. BENSOUSSAN, J. L. LIONS and G. PAPANICOLAOU, Asymptotic analysis for Periodic Structures. North-Holland, Amsterdam (1978).

[11] M. A. BIOT, Theory of propagation of elastic wares in a fluid. Saturated porous solid: I Low-frequency range. II Higher frequency range J.A.S.A 28, 168-191 (1956).

[12] C. BOUTIN, Dynamique des milieux poreux saturés déformables-Fonction de Green-Permeamètre dynamique These de Doctorat, Grenoble (1987).

[13] C. BOUTIN, G. BONNET and P. Y. BARD, Green functions and associated sources in infinite and stratified poroelastic media Geophys. J.R Ast-Soc. 90, 521-550 (1987).

[14] H. DI BENEDETTO, Modélisation du comportement des enrobés bitumineux et bitumes. Thèse de Docteur es-Sciences, Grenoble (1987).

[15] C. HUET, Etude par une méthode d'impédance du comportement des matériaux hydrocarbonés. Thèse de Docteur-Ingénieur, Paris (1963).

[16] F. H. GASKINS, J. G. BRODNYAN, F. F. WLADIMIR PHILIPPO and THELEN EDMUND, The rheology of Asphalt: II Flow characteristics of Asphalt. III Dynamic Mechanical Properties of Asphalt. Trans. Soc. rheology, IV, 265-296 (1960).

[17] F. GILBERT, Description des sols saturés par une méthode d'homogénéisation. Manuel de Rhéologie des Géomatériaux, pp. 317-333. Presses des Ponts \& Chaussées (1987).

[18] E. SANCHEZ PALENCIA, Non homogeneous media and vibration theory. Lectures Notes in Physics, Vol. 127. Springer-Verlag, Berlin (1980).

[19] E. SANCHEZ PALENCIA, Methode d'homogeneisation en théorie des suspensions et milieux composites. Conférence générale. 6ême Congrès Français de Mécanique, Lyon (1983).

[20] G. SAYEGH, Contribution à l'étude des propriétés viscoélastiques des bitumes purs et bétons bitumineux. Thèse de Docteur-Ingénieur, Paris (1965).

[21] G. V. VINAGRODOV, A. I. ISAYEU, V. A., ZOLDTAREV, and A. VEREBSKAYAGE, Rheological properties of road bitumen. Rheological Acta 16, 266-281 (1977). 